\title{
Standardized Echinacea purpurea extract primes an IFN specific innate immune monocyte response via JAK1-STAT1 dependent gene expression and epigenetic control of endogenous retroviral sequences
}

\section{Ken Declerck}

University Antwerp

Claudina Perez Novo

University Antwerp https://orcid.org/0000-0003-4562-4346

Lisa Grielens

University Antwerp

\section{Guy Van Camp}

University Antwerp https://orcid.org/0000-0001-5105-9000

\section{Andreas Suter}

A. Vogel Bioforce AG

Wim Vanden Berghe ( $\nabla$ wim.vandenberghe@uantwerpen.be )

Univesity Antwerp https://orcid.org/0000-0003-0161-7355

\section{Research Article}

Keywords: echinacea, interferon, monocyte, virus, kinase, chemokine, endogenous retroviral repeat, DNA methylation

Posted Date: May 15th, 2020

DOI: https://doi.org/10.21203/rs.3.rs-28886/v1

License: (c) (i) This work is licensed under a Creative Commons Attribution 4.0 International License.

Read Full License 


\section{Abstract}

Background: Herbal remedies of Echinacea purpurea tinctures are widely used today to reduce common cold respiratory tract infections.

Methods: A system biology approach involving genomewide transcriptome, epigenome and kinome activity profiles was applied to characterize the immunomodulatory effects of a standardized Echinacea

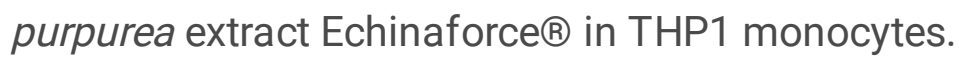

Results: Gene expression and DNA methylation analysis revealed activation of innate immunity related antiviral signaling networks, involving IFN, chemotaxis and immunometabolic pathways. Furthermore, phosphopeptide based kinome activity profiling and pharmacological inhibitor experiments with filgotinib confirm a key role for Janus Kinase (JAK)-1 dependent gene expression changes in innate immune signaling. Finally, Echinaforce ${ }^{\circledR}$ treatment induces DNA hypermethylation at intergenic $\mathrm{CpG}$, long/short interspersed nuclear DNA repeat elements (LINE, SINE) or long termininal DNA repeats (LTR). This changes transcription of flanking endogenous retroviral sequences (HERVs), involved in an evolutionary conserved (epi)genomic protective response against viral infections.

Conclusions: Altogether, our results suggest that Echinaforce ${ }^{\circledR}$ phytochemicals strengthen antiviral innate immunity by JAK1 responsive gene expression and epigenetic regulation of HERVs in monocytes, which supports its prophylactic use against common cold corona viruses (CoV), Severe Acute Respiratory Syndrome (SARS)-CoV, and new occurring strains such as SARS-CoV-2.

\section{Background}

Distinct species of the plant genus Echinacea have traditionally been used in North America against infectious diseases and wounds [1,2]. Currently, a wide variety of Echinacea preparations are used worldwide as complementary herbal remedy to improve the immune response to protect against common cold symptoms and influenza infections. Of all Echinacea species, Echinacea purpurea (purple coneflower) is the most popular variety used in Western countries.

Different Echinacea purpurea extracts (different species, plant parts, manufacturing) or derived compounds showed antioxidant, antibacterial, antifungal, antiviral and mosquitocidal activities in cell culture experiments [3], although absolute comparisons between studies with different preparations remain difficult $[4,5]$. A meta-analysis of six intervention studies with a total of 2458 participants revealed a significant $(P<0.0001)$ reduction of common cold symptoms, recurrence of respiratory tract infections and complications by the Echinacea treated versus the placebo control groups $[4,6]$.

Complex immunomodulatory actions of Echinacea have been described including both pro- and antiinflammatory effects $[2,3,7]$. The compounds that contribute to these activities are alkylamides, glycoproteins, polysaccharides and caffeic acid derivates that may act independently or in synergy [1, 3, 8, 9]. Therefore, different Echinacea preparations with variable constituents may lead to different immune 
outcomes [5]. For example, a polysaccharide-rich root extract increased the expression of MHC II, CD86 and CD54 markers on mice dendritic cells, while an alkylamide-rich leaf extract suppressed the expression of these surface markers [10].

In this study, we evaluated Echinaforce ${ }^{\circledR}$, a commercially registered herbal medicinal tincture of Echinacea purpurea plant (A.Vogel Bioforce, Switzerland) in several European countries including Switzerland, Austria, UK, Spain, Netherland, Denmark, Finland, Sweden, Slovenia as well as Canada. The tincture contains $5 \%$ root extract and $95 \%$ herb extract following extraction with $65 \%$ ethanol V/V. Echinaforce ${ }^{\circledR}$ phytochemicals reveal immune modulatory, anti-inflammatory, anti-bacterial, anti-viral and anti-parasitic activity [11-22]. Clinical efficacy could be shown with different batches in acute treatment [23] or for prevention [24] of respiratory tract infections. A 4-month randomized, double blind, placebocontrolled study ( $n=755$ subjects, of which 376 received placebo) on the safety and efficacy of Echinaforce ${ }^{\circledR}$ to prevent common cold symptoms, showed significantly less cold episodes and of shorter duration as well as lower infection recurrence rate in the Echinaforce ${ }^{\circledR}$ treated versus placebo treated group [24]. Moreover, no differences between placebo and Echinaforce ${ }^{\circledR}$ group were reported in relation to health risk and safety [24]. Despite the promising immune potentiating properties of Echinaforce ${ }^{\circledR}$, the responsible molecular targets have only partially been identified. For example, multiple studies demonstrate that Echinacea alkylamides exert their action partially through the cannabinoid receptor 2 (CB2) $[8,20,25]$. Alkylamides are structurally similar to endocannabinoids, and bind CB2 with a higher affinity compared to endogenous cannabinoids [25]. This action of alkylamides on CB2 was the mechanism behind the upregulation of TNF-a in primary monocytes after Echinaforce $₫$ treatment [20]. In addition, the cAMP, p38/MAPK and JNK signaling pathways, as well as NF-KB and ATF2/CREB1 transcription factors were found to be involved in the Echinacea-induced TNF-a expression [20].

Furthermore, a recent study showed that ex vivo blood stimulation with LPS after an 8-day oral Echinacea administration resulted in the induction of anti-inflammatory cytokines (IFN- $\gamma$, IL-8, IL-10 and MCP-1) only in subjects with low basal levels of these cytokines [11]. Interestingly, individuals with high basal levels of these cytokines didn't show any further induction. The same was seen in subjects with high stress levels or high susceptibility to cold infections, suggesting that Echinaforce ${ }^{\circledR}$ mainly (or only) enhances immune responses in immunocompromised subjects [11]. To further clarify its mode of action, we applied a system biology approach by integrating genomewide transcriptome, epigenome and kinome activity profiles of THP1 monocytes treated with Echinaforce ${ }^{\circledR}$.

\section{Methods}

\section{Cell lines and treatments}

Echinaforce ${ }^{\circledR}$ (batch nr. 040070, A. Vogel Bioforce AG, Roggwil, Switzerland) is a standardized preparation obtained by ethanol extraction of freshly harvested Echinacea purpurea herb and roots (95:5). Echinaforce ${ }^{\circledR}$ is marketed as a registered medicinal product and produced under conditions of Good Manufacturing Practice (GMP). Thus, a consistent quality for each produced batch is mandatory 
and equal to the requirements for an allopathic remedy. The composition of marker compounds like alkylamides (i.e. those compounds known to characterize this species of Echinacea) was described previously $[3,7,26]$. In contrast to pressed juice extracts, Echinaforce ${ }^{\circledR}$ extract does not contain polysaccharides which are known to stimulate the immune system nonspecifically [27-30]. The alcohol concentration of Echinaforce ${ }^{\circledR}$ tincture extract was $65 \% \mathrm{v} / \mathrm{v}$ and solvent controls have been included in all experimental in vitro experiments to rule out nonspecific effects. In addition, the preparation was free of detectable endotoxin as determined by means of a commercial assay kit with a lower limit of detection 0.1 unit/ml (Lonza Walkersville Inc., MD).

THP1 cells were grown in RPMI-1640 medium supplemented with glutamine, $10 \%$ heat inactivated Fetal Bovine Serum, $50 \mathrm{IU} / \mathrm{mL}$ Penicillin, $50 \mu \mathrm{g} / \mathrm{mL}$ Streptomycin, $10 \mathrm{mM}$ HEPES and $0.05 \mathrm{mM} \beta$ mercaptoethanol. Cells were treated with $1 \%$ Echinaforce ${ }^{\circledR}$ tincture versus ethanol solvent control. Each treatment condition consisted of six biological replicates.

\section{Genome-wide gene expression analysis}

\section{Sample preparation and microarray processing}

THP1 cells were treated for $48 \mathrm{~h}$ with $1 \%$ Echinaforce ${ }^{\circledR}$ or ethanol solvent control. RNA was isolated using the RNeasy mini kit (Qiagen) according to manufacturer's instructions. RNA concentration and purity was measured using the Nanodrop 1000 spectrophotometer (ThermoFischer, CA, USA). RNA integrity of each sample was checked using using the Experion Automated Electrophoresis System (Bio-Rad, MO, USA). Total RNA (500ng) was amplified using the Illumina TotalPrep RNA Amplification kit (Life Technologies, Carlsbad, CA, USA). Briefly, RNA was reverse transcribed using T7 oligo(dT) primers, after which biotinylated complementary or anti-sense RNA (CRNA) was synthesized through an in vitro transcription reaction. Then, $750 \mathrm{ng}$ of amplified cRNA was hybridized to a HumanHT12 beadchip array (Illumina, San Diego, CA, USA) and further incubated for 18 hours at $58^{\circ} \mathrm{C}$ in a hybridization oven under continuous rocking. After several consecutive washing steps, bead intensities were read on an Illumina iScan. Microarray data and raw gene expression intensities were preprocessed and analyzed using the beadarray $R$ package [31]. Intensities were quantile normalized and $\log _{2}$ transformed. Raw and normalized array data were uploaded to the Gene Expression Omnibus (GEO) database and have accession number: GSE117904. Probes with a P-detection value higher than 0.05 in at least six samples were removed. Also, probes annotated as "bad" and "no match" as described before [32] were not kept for further analysis. Differentially gene expression was performed using the limma R package [33]. P-values were corrected for multiple testing using the method of Benjamini and Hochberg. Probes with a log2 fold change higher than 0.4 and an adjusted p-value less than 0.05 were defined as significant and kept for further analysis [34]. The probes were annotated with gene information using the illuminaHumanv4.db annotation dataset [35]. The gene IDs of the significant Illumina expression probes were uploaded into the IPA software to find enriched biological pathways, diseases and networks. Metascape protein-proteininteraction enrichment analysis of differentially expressed genes was performed with the following databases: BioGrid, InWeb_IM, OmniPath [36]. The Molecular Complex Detection (MCODE) algorithm in 
Metascape identified densely connected network components and applies pathway and process enrichment analysis to each MCODE component independently. The three best-scoring terms by $\mathrm{p}$-value are retained as the functional description of the corresponding MCODE components (as shown in the tables).

\section{Quantitative realtime PCR}

To validate microarray data, THP1 cells were treated with 1\% Echinaforce ${ }^{\circledR}$ or Solvent for the indicated time-points $(3,6,12,24$ and $48 \mathrm{~h})$ in three independent experiments. The effect of JAK1 inhibition was determined by treating the cells with $1 \mu \mathrm{M}$ JAK1 inhibitor Filgotinib (GLPG0634, Selleckchem) for 30 min before adding Echinaforce $\AA^{\circledR}$. Total RNA was isolated using the RNeasy mini kit (Qiagen, Hilden, Germany) including a DNAse treatment step as suggested by the manufacturer. Then 750 ng RNA was reverse transcribed into cDNA using oligo dT (Invitrogen), M-MLV reverse transcriptase (Promega, Wisconsin USA), $2.5 \mathrm{mM}$ dNTPs and RNaseOUT (Invitrogen). Samples were incubated on $42^{\circ} \mathrm{C}$ for $60 \mathrm{~min}$ and $75^{\circ} \mathrm{C}$ for $15 \mathrm{~min}$. For the HERV genes, cDNA synthesis was performed using random primers (Invitrogen) and incubation of the samples at $37^{\circ} \mathrm{C}$ for $60 \mathrm{~min}$ and $75^{\circ} \mathrm{C}$ for $15 \mathrm{~min}$. qPCR was performed using the GoTaq qPCR Master Mix (Promega, Wisconsin USA) on a StepOnePlus Real-Time PCR machine (Applied Biosystems). Following primers were used: MX1 forward primer 5'-

GTTTCCGAAGTGGACATCGCA-3', MX1 reverse primer 5'-CTGCACAGGTTGTTCTCAGC-3'

(NM_001144925), IFITM1 forward primer 5'-CCAAGGTCCACCGTGATTAAC-3', IFITM1 reverse primer 5'ACCAGTTCAAGAAGAGGGTGTT-3' (NM_003641), STAT1 forward primer 5'- CCATCCTTTGGTACAACATGC3', STAT1 reverse primer 5'-TGCACATGGTGGAGTCAGG-3' (NM_007315), IL8 forward primer 5'-

GCTCTCTTGGCAGCCTTCCTGA-3', IL8 reverse primer 5'-ACAATAATTTCTGTGTTGGCGC-3' (NM_000584), CXCL10 forward primer 5'-GAAAGCAGTTAGCAAGGAAAGGT-3', CXLC10 reverse primer 5'-

GACATATACTCCATGTAGGGAAGTGA-3' (NM_001565), ACTB forward primer 5'-

CTGGAACGGTGAAGGTGACA-3', and ACTB reverse primer 5'- AAGGGACTTCCTGTAACAATGCA-3'

(NM_001101). Primer sequences for HERVs were derived from [37]. Each sample was ran in triplicate and the median Ct-values between each replicate group was selected. Ct-values were normalized using ACTB housekeeping gene. The ddCt-values or log fold changes (logFC) were calculated using the solvent control as reference sample. A paired t-test t-test was used to determine the significance of the differences between Echinaforce $₫$ and solvent expression levels.

\section{Kinase activity profiling}

\section{Sample preparation}

THP1 cells were treated with $1 \%$ Echinaforce ${ }^{\circledR}$ or ethanol solvent control for $15 \mathrm{~min}$. Cell lysates were prepared according to manufacturer's instructions. In short, cells were washed twice with cold 1X PBS and lysed with lysis buffer (1:100 dilution of Halt Phosphatase Inhibitor Cocktail and Halt Protease Inhibitor Cocktail EDTA free in M-PER Mammalian Extraction Buffer (ThermoFisher Scientific ${ }^{\mathrm{TM}}$, Rockford, USA) at a ratio of $100 \mu$ l buffer per $1 \times 10^{6}$ cells. Lysates were then incubated on ice for $15 \mathrm{~min}$ and 
centrifuged for 15 minutes at $16000 \mathrm{xg}$ at $4^{\circ} \mathrm{C}$. Protein concentration was quantified using the Pierce BCA Protein Assay Kit (ThermoFisher Scientific ${ }^{\mathrm{TM}}$, Rockford, USA).

\section{Serine/threonine kinases (STK) and tyrosine kinase (PTK) pamgene assay and data analysis}

Kinase activity profiling was performed PamChip ${ }^{\circledR}$ preprocessing and kinase activity profiling was performed according to manufacturer's instructions (PamGene International BV, 's-Hertogenbosch, The Netherlands). The first part of the protocol consisted in the blocking of the arrays with $2 \%$ BSA followed by several washing steps. Then $0.5 \mu \mathrm{g}$ for STK and $5 \mu \mathrm{g}$ for PTK assays together with the correspondent reaction mixes (purchased from the Pamgene) were loaded onto the arrays and incubated in the microarray system PamStation ${ }^{\circledR} 12$ instrument (PamGene International, Den Bosch, The Netherlands). In this step, the ATP contained in the mix leads to the activation of the kinases in the lysate which will result in the phosphorylation of the peptides on the array. Peptide phosphorylation intensities are then detected with the primary STK antibody mix and FITC-labeled antibody for STK assay and with the FITC-labelled PTK antibody (PTK assay). Images are then taken by the CCD camera in the PamStation ${ }^{\circledR} 12$ and processed by the Bionavigator software. Peptide intensities data were $\log _{2}$ transformed and differences in phosphorylation between Echinaforce ${ }^{\circledR}$ treated and control cultures were determined by using an univariate student t-test analysis corrected for multiple testing using the Benjamini and Hochberg method [34].

To identify potentially activated or inhibited kinases we used the STK or PTK Upstream Kinase analysis PamApp from the Bionavigator Software. The analysis is based on "in silico predictions" for the upstream kinases of phosphorylation sites in the human proteome that are retrieved from the phosphoNET database [38]. In short, a prediction algorithm is derived from known interactions between kinases and phosphorylation sites. The prediction algorithm is then used to predict the strength of undocumented interactions. The Bionavigator application uses PhosphoNet database to map putative kinases upstream of the phospho-peptides (a kinase can have multiple possible phosphosites, and a single site can be phosphorylated by different kinases). For each set of peptides mapped to a specific kinase, a "difference statistics" is calculated (=normalized kinase statistics) using following formula:

$$
\tau=\frac{1}{n} \sum_{i=1}^{n} \frac{\bar{p}_{i-}-\bar{p}_{i 2}}{\sqrt{s_{11}^{2}+s_{i 2}^{2}}} \text { with } \bar{p}_{i j} \text { and } \bar{s}_{i j}
$$

as the sample mean and variance of the intensity of peptide $\mathrm{i}$ in group $\mathrm{j}$, respectively, whereas $\mathrm{n}$ is the number of peptides linked with a specific kinase. A positive kinase statistic means that the kinase is activated, while a negative statistic means the kinase is inactivated compared to the control group. The kinases are subsequently ranked based on a specificity and significance score which are calculated using permutation of the peptides and samples, respectively. Following formula is used: ${ }^{Q=-\log _{10}\left(\max \left(\frac{m}{M}, \frac{1}{M}\right)\right) \text {, }}$ where $m$ is the number of times out of $M$ permutations that $\left|\tau_{p}\right|>|\tau|$, where $\tau_{p}$ is the value of the difference statistic obtained after permutation of the samples or peptides. The significance score represents the magnitude of the change represented by the normalized kinase statistic. The specificity score represents the specificity of the of normalized kinase statistic in terms of the set of peptides used for the corresponding kinase. The higher the score the less likely it is that the observed 
normalized kinase statistics could have been obtained using a random set of peptides from the data set. The sum of the significance and specificity score is used to rank the kinases [39].

\section{Genome-wide DNA methylation analysis}

\section{Sample preparation}

THP1 cells were cultured for $48 \mathrm{~h}$ with $1 \%$ Echinaforce ${ }^{\circledR}$ or ethanol solvent control. Corresponding cellular genomic DNA was isolated using the DNeasy Blood \& Tissue kit (Qiagen, Hilden, Germany) according to manufacturer's instructions. DNA concentration and purity was measured using the Nanodrop 100 spectrophotomer and $1 \mu \mathrm{g}$ of DNA was used for bisulfite conversion using the EZ DNA methylation Kit of Zymo Research according to manufacturer's instructions. Successful bisulfite conversion was checked using a methylation-specific PCR in a region of the SALL3 gene (see [40] for primer sequences).

\section{EPIC DNA methylation array}

The Infinium HumanMethylationEPIC BeadChip array (IIlumina, San Diego, CA, USA) was used to measure genome-wide DNA methylation. $4 \mu \mathrm{L}$ of bisulfite-converted DNA from each sample was amplified, fragmented, precipitated, resuspended and subsequently hybridized onto the BeadChips. After overnight incubation of the BeadChips, unhybridized fragments were washed away, while hybridized fragments were extended using fluorescent nucleotide bases. Finally, the BeadChips were scanned using the Illumina iScan system to obtain raw methylation intensities of each probe.

\section{EPIC DNA methylation data preprocessing and analysis}

The R package RnBeads was used to preprocess the Illumina 450K methylation data [41]. CpG-probes were filtered before normalization based on following criteria: probes containing a SNP within $3 \mathrm{bp}$ of the analyzed CpG site, bad quality probes based on an iterative greedycut algorithm with a detection $p$-value threshold of 0.01 , and probes with missing values in at least one sample. After filtering these CpG-probes, methylation values were within-array normalized using the beta mixture quantile dilation (BMIQ) method [42]. Another filtering step was performed after normalization based on following criteria: probes measuring methylation not at $\mathrm{CpG}$ sites (CC, $\mathrm{CAG}, \mathrm{CAH}, \ldots)$ and probes on sex chromosomes.

The methylation beta-values were transformed to $M$-values $\left(M=\log _{2}(\beta /(1-\beta))\right)$ prior to further analyses. The moderated t-test incorporated in the limma R package [33] was used to calculate the statistics and pvalues of the methylation differences between Echinaforce ${ }^{\circledR}$ - and solvent-treated samples. Significant differentially methylated probes (DMPs) were selected based on a false discovery rate (FDR) $<0.1$ and a difference in beta-value of at least 0.05. The DMPs were annotated with gene information using the IlluminaHumanMethylationEPICmanifest R package [43]. Further gene information was retrieved from the UCSC genome browser (human hg19). Enrichment of genomic regions was calculated using the Fisher's exact test. Pathway analysis of the genes harboring a DMP was performed using the Ingenuity Pathway 
Analysis (IPA) software. Raw and normalized array data were uploaded to the Gene Expression Omnibus (GEO) database and have accession number: GSE117904.

\section{Protein expression of Mx1, STAT1 and IFITM1 proteins using western blotting}

Protein expression levels of MX1, STAT1 and IFTIM1 were determined in THP1 cells treated with 1\% Echinaforce ${ }^{\circledR}$ or ethanol solvent control for 48 hours, as explained before. Then, cells were washed and incubated 15 minutes on ice in lysis buffer containing: $150 \mathrm{mM} \mathrm{NaCl}, 1 \mathrm{mM}$ EGTA, $1 \mathrm{mM}$ EDTA, $1 \mathrm{mM}$ ßglycerolphosphate, $1 \%$ Triton X-100 (w/v), 20mM Tris HCl, pH = 7.5 and proteinase inhibitor (cOmplete ${ }^{\mathrm{TM}}$, EDTA-free Protease Inhibitor Cocktail, Sigma-Aldrich, USA) plus PhosphataseArrest ${ }^{\text {TM }}$ Phosphatase Inhibitor Cocktail (phosphataseArrest ${ }^{\mathrm{TM}}$, G-Biosciences, USA). Cells were subsequently centrifuged for 15 minutes at $200 \mathrm{~g}$ at $4^{\circ} \mathrm{C}$ and supernatant containing the soluble proteins were stored at $-20^{\circ} \mathrm{C}$ until use. Protein lysates $(20 \mu \mathrm{g})$ were mixed with $5 \mathrm{X}$ sample buffer ( $5 \%$ SDS, $20 \%$ glycerol, $0.2 \%$ bromophenolblue, $250 \mathrm{mM} \mathrm{DTT}, 65 \mathrm{mM}$ Tris $\mathrm{HCl}$ ) all purchased from Sigma Aldrich (Missouri, USA), heated for 5 minutes at $95^{\circ} \mathrm{C}$ and loaded in a $12 \%$ SDS-PAGE gel. Proteins contained in the homogenates were separated during 30 minutes at $60-70 \mathrm{~V}$ and 1 hour at a constant voltage of $130 \mathrm{~V}$. Further, $10 \mathrm{ml}$ of BenchMark $^{\text {TM }}$ Pre-Stained Protein Standard (Life Technologies, CA, USA) was also loaded next to the samples. After separation proteins ttransferred onto a Nitrocellulose Membrane (BioRad, CA, USA) during 2 hours at $45 \mathrm{~V}$. Non-specific binding sites were blocked by incubating the membranes with blocking buffer $(0.05 \%$ Tween $20,1 \times$ TBS, $5 \%$ BSA) for 1 hour at room temperature. The membrane was then incubated with the primary antibodies: MX1 (D3W7I) Rabbit mAb \#37849, IFITM1 Antibody Rabbit pAb \#13126 and the STAT1 (42H3) Rabbit mAb \#9175 (all purchased from Cell Signaling Technology, Massachusetts, USA) or rabbit polyclonal Anti-GAPDH antibody (ab9485, Abcam, Cambridge, UK) overnight at $4^{\circ} \mathrm{C}$. After membranes were washed, they were incubated with (1:10000) Donkey anti-Rabbit IgG $(\mathrm{H}+\mathrm{L})$ Secondary Antibody-HRP (Thermo Fisher Scientific, Massachusetts, USA) for one hour at room temperature. Chemiluminiscence detection was performed using the ECL detection kit (Pierce ${ }^{\mathrm{TM}} \mathrm{ECL}$ Western Blotting Substrate (Thermo Fisher Scientific, Massachusetts, USA) in a ChemiDoc MP system (BioRad, CA, USA).

\section{Assessment of IL-8 and CXCL10 levels}

Cell culture supernatants were collected after 3, 6, 12, 24 and 48 hours and assayed for chemokines CXCL10 and IL8 by means of an enzyme-linked immunosorbent assay (ELISA) purchased from Invitrogen (CA, USA) following manufacturer's instructions. The assays have a detection limit of $2 \mathrm{pg} / \mathrm{ml}$ for CXCL10 and $5 \mathrm{pg} / \mathrm{mL}$ for IL-8.

\section{Results}

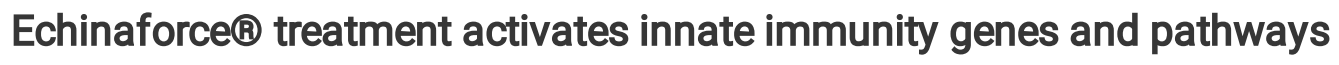

Widespread gene expression changes in monocyte THP1 cells were detected upon 48h 1\% Echinaforce ${ }^{\circledR}$ treatment. Based on significance criteria of FDR $<0.05$ and absolute $\log _{2}$ fold change $>0.4$, Echinaforce ${ }^{\circledR}$ 
induced modest upregulation of 205 expression probes (173 genes) while 124 probes ( 99 genes) were downregulated compared with the ethanol treated solvent controls (Figure 1A and Supplementary table 1). In contrast to pharmacological drugs (for example glucocorticoids (GC)) which can trigger drastic expression changes of GC-responsive genes (typically, $\log _{2}$ fold $>1$ ), many bioactive phytochemicals rather induce moderate transcriptional changes (typically $\log _{2}$ fold $>0,4$ ) of multiple genes converging on the same pathway [44-46]. Genes differentially expressed (DEG) by Echinaforce ${ }^{\circledR}$ treatment were enriched for IPA canonical pathways related to innate immune responses including interferon signaling, interferon regulatory factor (IRF) activation and the role of pattern recognition receptors, among others (Figure 1B-C and Supplementary table 2). Interestingly, most of these pathways were predicted to be activated, as can be seen from the highly positive activation z-scores. Interferon (IFN)-a/b and IFN-g both induce IFN-stimulated gene (ISG) expression through Janus kinase (JAK)-dependent phosphorylation of signal transducer and activator of transcription factors (STAT) 1 and STAT2 [47-52]. In line with the latter reports, we could observe transcriptional activation of various antiviral gatekeepers and interferon inducible proteins (i.e. MX1, IFI6/27/35/44, IFITM1/2/3, IFIT1/2/3, ISG15/20, IRF7/9), including various STAT1 target genes (Figure 1C, Supplementary Table 1-2). Logically, pathways related to viral infection and replication were predicted to be inhibited (activation z-score $<-2$ ). Also pathways involving cellular movement, migration, recruitment and chemotaxis were predicted to be activated (activation z-scores $>2$ ) (Figure 1D). Aside from ISGs, transcription of various chemokines and receptors (IL8, CXCL10, CXCL8, CCL2, CCL5, CXCR4, CCR2 as well as cannabinoid receptor GPR18) was also increased. In full accordance, recruitment and adhesion of immune cells, infection and immune related processes were found top ranked enriched diseases and biological functions in IPA analysis (Supplementary table 3).

Complementary to IPA analysis, protein-protein-interaction enrichment analysis of DEGs by STRING [53] and Metascape [36] algorithms was performed. This revealed strong enrichment of protein-protein interactions responding to a chemical stimulus, which triggers a defensive antiviral innate immune response involving IFN, TLR, NOD, RIG, cytokine, chemokine and NF-KB signaling pathways (Figure 2, Supplementary Table 4). More particularly, Metascape MCODE analysis identified 3 interconnected subnetworks in the antiviral cytokine response: cellular response to interferon, regulation of leukocyte chemotaxis and (mitochondrial) metabolism (Supplementary table 4).

To evaluate and validate the time dependent transcriptional dynamics of different genes involved in IFN and chemotaxis innate immune signaling observed in the microarray data, mRNA expression and protein levels for STAT1, MX1, IFITM1, CXCL8 and CXCL10 were measured using qPCR, ELISA and immunoblotting assays in THP1 monocytes following Echinaforce ${ }^{\circledR}$ treatment. Induction of STAT1 and the interferon-stimulated genes MX1 and IFITM1 expression could clearly be confirmed, with maximal mRNA transcription levels observed after 48h treatment (Figure 3A). Corresponding changes in STAT1 protein expression levels could also be detected by Western analysis (Figure 3B), whereas we failed to detect significant amounts of MX1 and IFITM1 protein (data not shown). Whether MX1 and IFITM1 protein expression is susceptible to additional posttranscriptional silencing mechanisms (miRNA, IncRNA) or posttranslational modifications which result in high turnover rates and low protein expression 
levels needs further investigation [54, 55]. For the chemokines IL8 and CXCL10, persistent gene induction could be observed until 48h, with peak transcription levels after $3 \mathrm{~h}$ (Figure $3 \mathrm{~A}$ ). Accumulation of these chemokines in the cell culture supernatants was also detected using ELISA technique and showed that the levels of both chemokines significantly increased with the incubation time of Echinaforce ${ }^{\circledR}$, as compared to control setups.

\section{Echinaforce $\circledast$ treatment activates JAK1, NF-KB and MAPK kinases}

To identify most important upstream kinase pathways responsible for gene expression changes in THP1 monocytes following Echinaforce ${ }^{\circledR}$ treatment, we performed a Pamchip kinome activity profiling assay [39]. This peptide array approach allows characterization of cellular serine/threonine or tyrosine kinome activity profiles following on chip in vitro kinase reaction of 144 conserved kinase consensus peptide motifs in presence of THP1 monocyte lysates left untreated or following Echinaforce ${ }^{\circledR}$ treatment [56-59]. Using the upstream kinase prediction tool of the Bionavigator PamGene software, the qualitative and quantitative changes in phosphopeptide chip intensities upon Echinaforce ${ }^{\circledR}$ treatment were translated into a pattern of activated or inhibited upstream kinases (Figure 4A and Supplementary table 5). In agreement with the transcriptional activation of the IFN signaling pathway described above (Figure 1C), Pamchip kinome profiling [39] revealed activation of the JAK1 kinase which is important in the phosphorylation of STAT kinases and subsequently downstream regulation of IFN-stimulated genes. Furthermore, in line with pathway analysis of transcriptome data, we also identified activation of the tyrosine kinase TEC (Figure 4B) (Supplementary table 2-4). Surprisingly, our analysis did not detect significant activity changes of early IFN kinases TBK1 and IKK [60].

Besides, we also identified various Echinaforce ${ }^{\circledR}$ activated kinases belonging to the MAPK superfamily of kinases: p38 MAPK (MAPK11, -12, -13, and -14), JNK (MAPK8, -9 and -10) and ERK1 (Figure 4C). This upstream regulators are also predicted by IPA to control various canonical pathways, including pattern recognition receptors in recognition of bacteria and viruses, activation of IRF by cytosolic pattern recognition receptors and role of MAPK signaling in the pathogenesis of influenza among others.

To further verify crucial involvement of JAK kinase activation in downstream gene expression effects upon Echinaforce ${ }^{\circledR}$ treatment, we compared THP1 gene expression changes following Echinaforce ${ }^{\circledR}$ treatment in presence or absence of the pharmacological JAK1 inhibitor filgotinib. We found that filgotinib significantly suppresses the Echinaforce ${ }^{\circledR}$ responsive genes MX1 and IFITM1, whereas STAT1, CXCL10 and IL8 gene expression were less significantly suppressed (Figure 4D). Altogether, experiments with the JAK1 inhibitor filgotinib strenghten our transcriptome and kinome data analysis, pointing to JAK1-specific regulation of downstream gene expression changes in response to Echinaforce ${ }^{\circledR}$ treatment.

\section{Echinaforce® treatment elicits epigenetic changes in innate immunity gene pathways}

Epigenetics seems to be important during monocyte differentiation and in the immunological memory of macrophages $[61,62]$. Today, various bioactive phytochemicals have been identified which modulate 
inflammation through epigenetic reprogramming $[63,64]$. Different phytochemicals and nutrients are known to change DNA methylation and histone modifications by directly influencing epigenetic enzymes or by interfering with the availability of the substrates/cofactors of these enzymes [65-67]. To assess whether the Echinaforce ${ }^{\circledR}$ induced changes in transcriptome profiles in THP1 cells are associated with DNA methylation changes, we measured complementary changes in DNA methylation profiles using the Illumina EPIC methylation array. Significant DNA methylation changes were observed following $48 \mathrm{~h}$ exposure to Echinaforce ${ }^{\circledR}$ (Figure 5A and Supplementary table 6).

A total of $1,875 \mathrm{CpG}$ sites was found differentially methylated $(\mathrm{FDR}<0.1)$ with a methylation difference of at least $5 \%$. Typically, DNA methylation changes after short (24-72h) exposure to phytochemicals and nutrients are much smaller than cancer associated DNA methylation changes in oncogenes or tumor suppressor genes which accumulate for many years in response to the microenvironment $[46,68,69]$. However, similar DMR effects sizes and cutoff $(<5 \%)$ were found to be biologically meaningful in various disease etiologies $[40,70,71]$.

From the $1,875 \mathrm{CpG}$ sites identified, only 40 differentially methylated positions (DMPs) were hypomethylated whereas 1,835 DMPs were hypermethylated. DMPs were mainly enriched in gene bodies, intergenic, and $\mathrm{CpG}$-poor regions, while depleted in $\mathrm{CpG}$ islands, promoter, and enhancer regions (Figure 5B). Only 1,259 of the 1,875 CpG-probes (67\%) were located in a gene or 1,500 bp upstream of a gene. Similarly, DNA methylation variation in the immune system was predominantly found at at $\mathrm{CpG}$ islands within gene bodies, which have the properties of cell type-restricted promoters, but infrequently at annotated gene promoters or CGI flanking sequences (CGI "shores") [72]. Subsequent IPA pathway enrichment analysis of the genes containing DMPs revealed inflammation or immunological diseases among others (Supplementary Table 6). Of particular interest, one of the top enriched pathways ('Superpathway of Inositol Phosphate Compounds') controls various epigenetic processes related to the interferon response [73-75].

Since both gene expression and kinase profiling both revealed the involvement of interferon signaling pathways, we also checked whether methylation of IFN pathway genes was affected by Echinaforce ${ }^{\circledR}$ treatment. Eight probes located in BCL2, JAK1, STAT1, PIAS1 and TAP1 did show an FDR < 0.1, with small methylation differences (between 1 and $3 \%$ ) (Figure 5C). Whether these small methylation changes are sufficient to finetune the immune gene response needs further investigation.

Since most of the DMPs were located in intergenic regions and gene bodies, only a small subset of genes containing a DMP also resulted in a significant change in gene expression (Figure 5D). Only seven genes were both differentially methylated and expressed, based on the significance criteria described above: i.e. Calsyntenin 2 (CLSTN2), Enhancer Of Zeste 2 Polycomb Repressive Complex 2 Subunit (EZH2), Growth arrest-specific protein (GAS)-7, neuron navigator (NAV)-3, Thioredoxin Reductase (TXNRD)-1, Tryptophanyl-tRNA synthetase (WARS) and Zinc Finger Transcription Factor (ZNF)-644. When using less stringent significance criteria, leaving out the effect size cutoff (logFC), 574 CpG site - gene pairs were found to be differentially expressed and methylated. Upon further comparing canonical pathways which 
are significantly enriched for both lists of differentially expressed genes and the list of differentially methylated genes, we identified 10 common biological processes (Figure 5E). Remarkably, common pathways include NF-KB signaling (NF-KB activation by viruses, NF-KB signaling), MAPK signaling (LPSstimulated MAPK signaling, UVA-induced MAPK signaling), and immune responses (i.e. Role of pattern recognition receptors in recognition of bacteria and viruses, Role of NFAT in regulation of the immune response, phagosome formation, CD40 signaling, leukocyte extravasation signaling).

\section{Echinaforce ${ }^{\circledR}$ treatment changes DNA repeat methylation and HERV transcription levels}

DNA repeats and transposons require hypermethylation to maintain genomic instability and prevent transposition [76-80]. Interestingly, differentially methylated probes (DMPs) demonstrated a considerable enrichment in LINE, SINE and LTR transposon repeats, flanking endogenous retroviral sequences (HERVs) (Figure 6A-B). This DMPs decreased transcription of MER4D, MER57B1, MLT1C627, MLT2B4 HERVs after 12 and 48h Echinaforce ${ }^{\circledR}$ treatment, whereas MLT1B and MLT1C49 HERVs were only transiently repressed at $12 \mathrm{~h}$ (Figure 6C). However, it remains unclear whether innate immune signaling (IFN response, chemotaxis, and immunometabolism) is driving HERV regulation or vice versa to mediate viral protection.

\section{Discussion}

In this study, we applied for the first time a systems biology approach to characterize a possible mode of action of a standardized medicinal Echinacea purpurea tincture Echinaforce ${ }^{\circledR}$, which is widely used as a herbal remedy against respiratory tract infections. Microarray, QPCR, Western and ELISA based assays demonstrate that treatment of THP1 monocyte cells with Echinaforce ${ }^{\circledR}$ phytochemicals elicit time dependent gene expression changes in innate immunity signaling networks, involving the IFN (MX1, IFITM1, STAT1, STAT2) chemotaxis (IL8, CXCL10) and immunometabolic (ISG15, PKM2, SQSTM1) pathway.

Most cells express a set of membrane and cytoplasmic receptors to detect viral RNA and DNA molecules: Pattern Recognition Receptors (PRRs). These receptors control innate immune signaling to activate the synthesis of interferons during a viral infection. In addition to pathogens, autophagy, metabolic and chemical stress, DNA damage, unfolded protein response, can also regulate innate immunity through cellautonomous responses. Either IFN-inducible or constitutive, these processes aim to guarantee cell homeostasis or a biodefense mechanism against (non-self) hazardous molecules [81]. Of importance, these distinct constitutive cell-autonomous responses appear to be interconnected and can also be modulated by microbes, viruses and PRRs [82]. Our results suggest that Echinaforce ${ }^{\circledR}$ phytochemicals prime innate immunity pathways via activation of interferon and chemokine gene expression. As such, secondary metabolite phytochemicals involved in plant immunity may train evolutionary conserved innate immune responses across species [83-85]. For example, transcriptional upregulation of the protein kinase receptor (PKR, EIF2AK2), a cytoplasmatic pattern-recognition receptor could be observed. PKR is known to transduce RNA helicase (MDA5) dependent virus signals for type I IFN induction [86]. Interferon 
regulatory factor 7 (IRF7) is another key protein found strongly upregulated. Transcription factors IRF7 together with IRF3 regulate expression of early type I IFN and other proteins involved in the innate antiviral immune response (activation of IRF by cytosolic pattern recognition receptors) [87] (Supplementary tables 1-2-3-4). Signal transduction via PKR occurs mainly via NF-KB and MAPK pathways (Role of PKR in Interferon induction and antiviral response) [88]. Another important intracellular pattern-recognition receptor for viral RNA which was found to be upregulated by Echinaforce $\AA$ was the RNA helicase MDA5 (IFIH1) [60]. Furthermore, upregulation of the NF-KB subunits RelB and NFKB2/p52 was observed, which can promote downstream production of innate immunity chemokines (NF-KB activation by viruses, NF-KB signaling) [89].

In line with our results which show activation of innate immunity and inhibition of viral infection and replication gene responses, anti-viral effects against influenza infection and activation of IFN pathways have also been reported in vivo following Echinaforce ${ }^{\circledR}$ tincture treatment $[11,15,21]$. Our in vitro results are also in line with observations in human studies ex vivo/in vivo showing increased immunomodulating as well as chemotactic neutrophil effects following Echinaforce ${ }^{\circledR}$ treatment, especially in immunocompromised people $[11,23,24,90]$. For example, the antiviral ability of CXCL 10 has been attributed to its chemoattractant effects which promote recruitment of neutrophils [91-94]. The latter illustrates that both neutrophils and inflammatory monocytes are intertwined in the immune system's anti-viral response [95]. Similar results were previously obtained in murine dendritic cells, illustrating that Echinaforce ${ }^{\circledR}$ stimulates cell mobility and chemotaxis and alters expression of cell adhesion and motility genes [96]. Other studies showed that Echinaforce ${ }^{\circledR}$ may reverse the chemokine induction of virus-infected cells [12, 97-99]. Paradoxically, Echinaforce ${ }^{\circledR}$ may induce cytokine and chemokine expression in uninfected cells, but suppress their expression upon virus infection or LPS stimulation [30, 97-99]. Similarly, Echinaforce ${ }^{\circledR}$ increased the transcription of TNF- $a$ in human monocytes, but reduced the LPS-stimulated TNF-a protein production [20]. Although studies suggest that this stimulatory effect may be the result of bacterial-derived LPS and lipoproteins [27-30], our Echinaforce ${ }^{\circledR}$ tincture contains no polysaccharides, neither endotoxins. Altogether, the latter suggests that its immunomodulatory effects are due to the active compounds present in the formulation $[13,20]$. Similar activation of IFN innate immunity and viral protection has been observed in presence of avocado and apple extract $[100,101]$. Interestingly, in the latter case, effects were attributed to oligomeric proanthocyanidins and lost with their monomeric form [101].

With respect to immunometabolism, mitochondrial metabolism shows a remarkable sensitivity to chemokine and IFN signaling $[95,102]$. For example, ISG15 is an interferon-stimulated, ubiquitin-like protein which regulates mitochondrial homeostasis and targets various proteins involved in catabolic autophagy metabolism in the mitochondria (mitophagy) during infection [103, 104]. Moreover, mitochondrial changes in immunometabolism (glycolysis, the tricarboxylic acid (TCA) cycle, the pentose phosphate pathway, fatty acid oxidation, fatty acid synthesis and amino acid metabolism) strongly contribute in (re)shaping immunity and production of neutrophil extracellular traps (NETs) [105-108]. 
Next, phosphopeptide based kinome activity analysis revealed Echinaforce ${ }^{\circledR}$ specific activation of innate immunity and IFN signaling via multiple kinases, including JAK1, TEC, p38 MAPK (MAPK11, -12, -13, and -14), JNK (MAPK8, -9 and -10) and ERK1 kinases $[109,110]$. JNK-STAT1 signaling induces various IFN responsive genes [111]. Moreover, JAK1 dependent regulation of downstream IFN and chemokine related gene expression after Echinaforce ${ }^{\circledR}$ treatment, could be reversed with the specific pharmacological JAK1 inhibitor filgotinib. TEC activation has important roles during innate immunity, i.e. IFN signaling via phosphorylation of JAK1 and JAK2 [109, 110], TLR signaling [112], assembly and activation of the caspase-8 inflammasome [113], macrophage survival [114], IL-8 production [115], phagocytosis [116], and NF-kB signaling [117]. p38 MAPK activation is involved in RIG-I dependent IFN signaling [118]. Various studies confirm involvement of these kinases in Echinacea biological action [20, 96, 119-122]. Alkylamides in the Echinaforce ${ }^{\circledR}$ tincture were found to be responsible for MAPK effects upon binding to CB2 receptors leading to increased CAMP, P38/MAPK and JNK signaling, NF-KB and ATF-2/CREB-1 activation [20]. Similarly, lipophilic extracts of Echinacea promoted murine dendritic cell maturation and mobility via the modulation of JNK, P38 MAPK and NF-KB pathways $[96,119,120]$. Another study demonstrated a Jak-Stat1 dependent antiviral response of Tripterygium wilfordii (Thunder of God Vine) via the quinone methide triterpene celastrol [123].

Finally, genomewide epigenetic analysis of DNA methylation changes following Echinaforce ${ }^{\circledR}$ treatment revealed almost 2000 DMP, enriched for immune disease and immunological pathways. Although the observed methylation changes are relatively small after $72 \mathrm{~h}$ treatment, cumulative effects can contribute in building an immune memory response by priming chromatin to mount faster and higher innate immune transcription upon re-stimulation of immune cells [84]. Besides the regulation of gene expression, DNA methylation is also involved in regulating alternative splicing, intron retention or promote cryptic transcription of non-annotated TSSs (TINATs) encoding immunogenic peptides which might prime an antiviral innate immune response [124-127]. As such, it appears that Echinaforce ${ }^{\circledR}$ treatment predominantly promotes epigenetic changes in innate immunity gene pathways and to a less extent of adaptive immune response genes.

Besides, the higher global DNA hypermethylation observed after Echinaforce ${ }^{\circledR}$ treatment in LINE, SINE and LTR transposon repeats flanking endogenous retroviral sequences (HERVs), may be part evolutionary conserved (epi)genomic protective response against retrotransposition and viral infection [128, 129]. Similarly, IFN was shown to promote DNA methylation silencing of repeats and noncoding RNAs [37, 128, $130,131]$. Specific HERVs have been proposed to establish a protective effect against exogenous viral infections [132]. HERVs can act as IFN-inducible enhancers and have shaped the evolution of a transcriptional network underlying the IFN response [132-135]. Of particular interest, the MER41B family of ERV sequences contains a STAT1 binding site and regulates expression of IFN- $\gamma$-responsive genes, such as absent in melanoma 2 (AIM2), and IFI6 $[136,137]$. CRISPR-Cas9 deletion of a subset of these HERV elements in the human genome impaired expression of adjacent IFN-induced genes and revealed their involvement in the regulation of essential immune functions, including activation of the AIM2 inflammasome. Along the same line, DNA methylation inhibitors trigger an IFN response through viral mimicry via transcription of dsRNAs of repetitive elements from HERVs which can activate RIG-I and 
MDA5 PRRs. RNA transcripts of HERVs can be reverse transcribed to generate ssDNA or expressed to generate proteins with viral signatures, much like the pathogen-associated molecular patterns of exogenous viruses, which allows them to be detected by the innate immune system $[138,139]$. In another example, silencing of the MLT1C49 HERV decreased expression of CXCL10 and CCL2 chemokines [140]. Finally, transcriptional changes of MLT1B and MER4D HERV transcription and innate immune signaling have also been described upon immunometabolic mitochondrial changes in protein kinase (PK)-M2 activity, which were counteracted by NFkB RelB [141]. From these examples, it appears that HERV regulatory sequences now constitute a dynamic reservoir of IFN-inducible enhancers fueling genetic innovation in mammalian immune defenses $[136,142,143]$.

Previous studies showed that Echinaforce ${ }^{\circledR}$, besides its immunomodulating activities is also very active as a virucidal agent against viruses with membranes, i.e. HSV-1, respiratory syncytial virus, all tested human and avian strains of influenza A virus, as well as influenza B virus [144]. Along the same line, Echinacea polyphenol quercetin was found to inhibit SARS-CoV entry in the host through binding SARSCoV-S2 protein [145]. Similarly, protective effects could recently also be observed in a reconstituted nasal epithelium cell culture system by exposing Echinaforce ${ }^{\circledR}$-treated respiratory epithelium to droplets of HCoV-229E, SARS- or MERS-CoVs, imitating a natural infection [146]. In contrast Echinaforce ${ }^{\circledR}$ was found to be less effective against intracellular virus replication. Consequently, virus already present within a cell could be refractory to the inhibitory effect of Echinaforce ${ }^{\circledR}$, but virus particles shed into the extracellular fluids would be vulnerable. Therefore the antiviral actions of the Echinaforce ${ }^{\circledR}$ may especially manifest during initial contact with the virus, i.e. at the inception of infection, and also during transmission of virus from infected cells.

In conclusion, our systems biology approach revealed that Echinaforce ${ }^{\circledR}$ phytochemicals trigger multiple antiviral immunemodulating bio-activities. Further studies in preclinical respiratory infection models and placebo controlled intervention studies are needed to identify discrete compounds or mixtures, which could be effective in prophylactic use against common cold corona viruses (CoV), Severe Acute Respiratory Syndrome (SARS)-CoV, and new occurring strains such as SARS-CoV-2.

\section{Declarations}

\section{Acknowledgements}

We thank Bioforce group Dr. A. Vogel (Roggswil, Switzerland) for providing Echinaforce ${ }^{\circledR}$ reagents

\section{Funding}

KD, CPN and WVB are supported by FWO grants (G079614N, G059713N) and BOF NOI/DOCPRO/GOA grants (UA, FFP140094).

\section{Ethics approval and consent to participate}

Not applicable 


\section{Consent for publication}

Not applicable.

\section{Availability of data}

Data are available on request. Transcriptome and DNA methylation data will be deposited in the public available GEO profile database.

\section{Competing interests}

Author Andreas Suter was employed by the company A. Vogel Bioforce AG, Roggwil, Switzerland. The remaining authors declare that the research was conducted in the absence of any commercial or financial relationships that could be construed as a potential conflict of interest.

\section{Authors' contributions}

$\mathrm{KD}, \mathrm{WVB}$ and $\mathrm{AS}$ conceived and designed the study. KD, CPN, LG performed the experiments and statistical data analysis. KD, CPN, LG AS and WVB interpreted the data. AS and GVC contributed reagents, EPIC array services and analysis tools. All authors wrote, evaluated revised and approved the final manuscript prior to submission.

\section{Supplementary Information}

Supplementary table 1: Differentially expressed probes $(F D R<0.05$ and $\log F C>0.4)$ after Echinaforce ${ }^{\circledR}$ tincture treatment.

Supplementary table 2: Enriched Ingenuity canonical pathways of differentially expressed genes after Echinaforce ${ }^{\circledR}$ tincture treatment.

Supplementary table 3: Ingenuity pathway enrichment analysis of diseases and biological functions of differentially expressed genes after Echinaforce ${ }^{\circledR}$ tincture treatment.

Supplementary table 4: STRING protein-protein-interaction plot and Metascape protein-protein-interaction MCODE network enrichment analysis of differentially expressed genes after Echinaforce ${ }^{\circledR}$ tincture treatment.

Supplementary table 5: PamGene upstream kinase analysis.

Supplementary table 6: Differentially methylated positions (FDR $<0.05$ and $\mid$ DeltaBetas $\mid>0.05)$.

Supplementary table 7: Enriched Ingenuity canonical pathways of differentially methylated genes after Echinaforce ${ }^{\circledR}$ tincture treatment. 


\section{References}

1. Barnes, J., et al., Echinacea species (Echinacea angustifolia (DC.) Hell., Echinacea pallida (Nutt.) Nutt.,Echinacea purpurea (L.) Moench): a review of their chemistry, pharmacology and clinical properties. J Pharm Pharmacol, 2005. 57(8): p. 929-54.

2. Barrett, B., Medicinal properties of Echinacea: a critical review. Phytomedicine, 2003. 10(1): p. 66-86.

3. Manayi, A., M. Vazirian, and S. Saeidnia, Echinacea purpurea: Pharmacology, phytochemistry and analysis methods. Pharmacogn Rev, 2015. 9(17): p. 63-72.

4. Karsch-Volk, M., B. Barrett, and K. Linde, Echinacea for preventing and treating the common cold. JAMA, 2015. 313(6): p. 618-9.

5. Binns, S.E., et al., Phytochemical variation in echinacea from roots and flowerheads of wild and cultivated populations. J Agric Food Chem, 2002. 50(13): p. 3673-87.

6. Schapowal, A., P. Klein, and S.L. Johnston, Echinacea reduces the risk of recurrent respiratory tract infections and complications: a meta-analysis of randomized controlled trials. Adv Ther, 2015. 32(3): p. 187-200.

7. Hudson, J.B., Applications of the phytomedicine Echinacea purpurea (Purple Coneflower) in infectious diseases. J Biomed Biotechnol, 2012. 2012: p. 769896.

8. Chicca, A., et al., Synergistic immunomopharmacological effects of $\mathrm{N}$-alkylamides in Echinacea purpurea herbal extracts. Int Immunopharmacol, 2009. 9(7-8): p. 850-8.

9. Dalby-Brown, L., et al., Synergistic antioxidative effects of alkamides, caffeic acid derivatives, and polysaccharide fractions from Echinacea purpurea on in vitro oxidation of human low-density lipoproteins. J Agric Food Chem, 2005. 53(24): p. 9413-23.

10. Benson, J.M., et al., Echinacea purpurea extracts modulate murine dendritic cell fate and function. Food Chem Toxicol, 2010. 48(5): p. 1170-7.

11. Ritchie, M.R., et al., Effects of Echinaforce(R) treatment on ex vivo-stimulated blood cells. Phytomedicine, 2011. 18(10): p. 826-31.

12. Sharma, M., et al., Induction of multiple pro-inflammatory cytokines by respiratory viruses and reversal by standardized Echinacea, a potent antiviral herbal extract. Antiviral Res, 2009. 83(2): p. 165-70.

13. Sharma, S.M., et al., Bactericidal and anti-inflammatory properties of a standardized Echinacea extract (Echinaforce): dual actions against respiratory bacteria. Phytomedicine, 2010. 17(8-9): p. 5638.

14. Sharma, M., R. Schoop, and J.B. Hudson, Echinacea as an antiinflammatory agent: the influence of physiologically relevant parameters. Phytother Res, 2009. 23(6): p. 863-7.

15. Pleschka, S., et al., Anti-viral properties and mode of action of standardized Echinacea purpurea extract against highly pathogenic avian influenza virus (H5N1, H7N7) and swine-origin H1N1 (S-OIV). Virol J, 2009. 6: p. 197. 
16. Sharma, M., R. Schoop, and J.B. Hudson, The efficacy of Echinacea in a 3-D tissue model of human airway epithelium. Phytother Res, 2010. 24(6): p. 900-4.

17. Canlas, J., et al., Echinacea and trypanasomatid parasite interactions: growth-inhibitory and antiinflammatory effects of Echinacea. Pharm Biol, 2010. 48(9): p. 1047-52.

18. Woelkart, K., et al., Bioavailability and pharmacokinetics of Echinacea purpurea preparations and their interaction with the immune system. Int J Clin Pharmacol Ther, 2006. 44(9): p. 401-8.

19. Sharma, M., et al., The potential use of Echinacea in acne: control of Propionibacterium acnes growth and inflammation. Phytother Res, 2011. 25(4): p. 517-21.

20. Gertsch, J., et al., Echinacea alkylamides modulate TNF-alpha gene expression via cannabinoid receptor CB2 and multiple signal transduction pathways. FEBS Lett, 2004. 577(3): p. 563-9.

21. Vimalanathan, S., et al., Prevention of influenza virus induced bacterial superinfection by standardized Echinacea purpurea, via regulation of surface receptor expression in human bronchial epithelial cells. Virus Res, 2017. 233: p. 51-59.

22. Vimalanathan, S., R. Schoop, and J. Hudson, High-potency Anti-influenza Therapy by a Combination of Echinacea purpurea fresh herb and root tinctures. Journal of Applied Pharmaceutical Science 2013. 3: p. 1-5.

23. Brinkeborn, R.M., D.V. Shah, and F.H. Degenring, Echinaforce and other Echinacea fresh plant preparations in the treatment of the common cold. A randomized, placebo controlled, double-blind clinical trial. Phytomedicine, 1999. 6(1): p. 1-6.

24. Jawad, M., et al., Safety and Efficacy Profile of Echinacea purpurea to Prevent Common Cold Episodes: A Randomized, Double-Blind, Placebo-Controlled Trial. Evid Based Complement Alternat Med, 2012. 2012: p. 841315.

25. Raduner, S., et al., Alkylamides from Echinacea are a new class of cannabinomimetics. Cannabinoid type 2 receptor-dependent and -independent immunomodulatory effects. J Biol Chem, 2006. 281(20): p. $14192-206$.

26. Bruni, R., et al., Analytical methods for the study of bioactive compounds from medicinally used Echinacea species. J Pharm Biomed Anal, 2018. 160: p. 443-477.

27. Pugh, N.D., C.R. Jackson, and D.S. Pasco, Total bacterial load within Echinacea purpurea, determined using a new PCR-based quantification method, is correlated with LPS levels and in vitro macrophage activity. Planta Med, 2013. 79(1): p. 9-14.

28. Pugh, N.D., et al., The majority of in vitro macrophage activation exhibited by extracts of some immune enhancing botanicals is due to bacterial lipoproteins and lipopolysaccharides. Int Immunopharmacol, 2008. 8(7): p. 1023-32.

29. Tamta, H., et al., Variability in in vitro macrophage activation by commercially diverse bulk echinacea plant material is predominantly due to bacterial lipoproteins and lipopolysaccharides. J Agric Food Chem, 2008. 56(22): p. 10552-6.

30. Todd, D.A., et al., Ethanolic Echinacea purpurea Extracts Contain a Mixture of Cytokine-Suppressive and Cytokine-Inducing Compounds, Including Some That Originate from Endophytic Bacteria. PLoS 
One, 2015. 10(5): p. e0124276.

31. Dunning, M.J., et al., beadarray: $R$ classes and methods for Illumina bead-based data. Bioinformatics, 2007. 23(16): p. 2183-4.

32. Barbosa-Morais, N.L., et al., A re-annotation pipeline for Illumina BeadArrays: improving the interpretation of gene expression data. Nucleic Acids Res, 2010. 38(3): p. e17.

33. Ritchie, M.E., et al., limma powers differential expression analyses for RNA-sequencing and microarray studies. Nucleic Acids Res, 2015. 43(7): p. e47.

34. Reiner, A., D. Yekutieli, and Y. Benjamini, Identifying differentially expressed genes using false discovery rate controlling procedures. Bioinformatics, 2003. 19(3): p. 368-75.

35. Dunning M, L.A.a.E.M., illuminaHumanv4.db: Illumina HumanHT12v4 annotation data (chip illuminaHumanv4). 2015, R package version 1.26.0.

36. Zhou, Y., et al., Metascape provides a biologist-oriented resource for the analysis of systems-level datasets. Nat Commun, 2019. 10(1): p. 1523.

37. Roulois, D., et al., DNA-Demethylating Agents Target Colorectal Cancer Cells by Inducing Viral Mimicry by Endogenous Transcripts. Cell, 2015. 162(5): p. 961-73.

38. Safaei, J., et al., Prediction of 492 human protein kinase substrate specificities. Proteome Sci, 2011. 9 Suppl 1: p. S6.

39. Chirumamilla, C.S., et al., Profiling Activity of Cellular Kinases in Migrating T-Cells. Methods Mol Biol, 2019. 1930: p. 99-113.

40. Szarc Vel Szic, K., et al., Epigenetic silencing of triple negative breast cancer hallmarks by Withaferin A. Oncotarget, 2017. 8(25): p. 40434-40453.

41. Assenov, Y., et al., Comprehensive analysis of DNA methylation data with RnBeads. Nat Methods, 2014. 11(11): p. 1138-1140.

42. Teschendorff, A.E., et al., A beta-mixture quantile normalization method for correcting probe design bias in Illumina Infinium 450 k DNA methylation data. Bioinformatics, 2013. 29(2): p. 189-96.

43. Hansen KD, IlluminaHumanMethylationEPICmanifest: Manifest for Illumina's EPIC methylation arrays. 2016, R package version 0.3.0.

44. Milenkovic, D., et al., Dietary flavanols modulate the transcription of genes associated with cardiovascular pathology without changes in their DNA methylation state. PLoS One, 2014. 9(4): p. e95527.

45. Chirumamilla, C.S., et al., Selective Glucocorticoid Receptor Properties of GSK866 Analogs with Cysteine Reactive Warheads. Front Immunol, 2017. 8: p. 1324.

46. Milenkovic, D., et al., A systems biology network analysis of nutri(epi)genomic changes in endothelial cells exposed to epicatechin metabolites. Sci Rep, 2018. 8(1): p. 15487.

47. Michalska, A., et al., A Positive Feedback Amplifier Circuit That Regulates Interferon (IFN)-Stimulated Gene Expression and Controls Type I and Type II IFN Responses. Front Immunol, 2018. 9: p. 1135. 
48. Mogensen, T.H., IRF and STAT Transcription Factors - From Basic Biology to Roles in Infection, Protective Immunity, and Primary Immunodeficiencies. Front Immunol, 2018. 9: p. 3047.

49. Verhelst, J., et al., Interferon-inducible protein $M x 1$ inhibits influenza virus by interfering with functional viral ribonucleoprotein complex assembly. J Virol, 2012. 86(24): p. 13445-55.

50. Verhelst, J., P. Hulpiau, and X. Saelens, Mx proteins: antiviral gatekeepers that restrain the uninvited. Microbiol Mol Biol Rev, 2013. 77(4): p. 551-66.

51. Smith, S.E., et al., Interferon-Induced Transmembrane Protein 1 Restricts Replication of Viruses That Enter Cells via the Plasma Membrane. J Virol, 2019. 93(6).

52. Bailey, C.C., et al., IFITM-Family Proteins: The Cell's First Line of Antiviral Defense. Annu Rev Virol, 2014. 1: p. 261-283.

53. Szklarczyk, D., et al., STRING v11: protein-protein association networks with increased coverage, supporting functional discovery in genome-wide experimental datasets. Nucleic Acids Res, 2019. 47(D1): p. D607-D613.

54. Hussein, H.A.M. and S.M. Akula, miRNA-36 inhibits KSHV, EBV, HSV-2 infection of cells via stifling expression of interferon induced transmembrane protein 1 (IFITM1). Sci Rep, 2017. 7(1): p. 17972.

55. Brogaard, L., et al., Late regulation of immune genes and microRNAs in circulating leukocytes in a pig model of influenza A (H1N2) infection. Sci Rep, 2016. 6: p. 21812.

56. Arsenault, R., P. Griebel, and S. Napper, Peptide arrays for kinome analysis: new opportunities and remaining challenges. Proteomics, 2011. 11(24): p. 4595-609.

57. Dussaq, A., et al., Mechanistic Parameterization of the Kinomic Signal in Peptide Arrays. J Proteomics Bioinform, 2016. 9(5): p. 151-157.

58. Labots, M., et al., Evaluation of a tyrosine kinase peptide microarray for tyrosine kinase inhibitor therapy selection in cancer. Exp Mol Med, 2016. 48(12): p. e279.

59. Baharani, A., et al., Technological advances for interrogating the human kinome. Biochem Soc Trans, 2017. 45(1): p. 65-77.

60. Mesev, E.V., R.A. LeDesma, and A. Ploss, Decoding type I and III interferon signalling during viral infection. Nat Microbiol, 2019. 4(6): p. 914-924.

61. Novakovic, B., et al., beta-Glucan Reverses the Epigenetic State of LPS-Induced Immunological Tolerance. Cell, 2016. 167(5): p. 1354-1368 e14.

62. Saeed, S., et al., Epigenetic programming of monocyte-to-macrophage differentiation and trained innate immunity. Science, 2014. 345(6204): p. 1251086.

63. Szarc vel Szic, K., et al., From inflammaging to healthy aging by dietary lifestyle choices: is epigenetics the key to personalized nutrition? Clin Epigenetics, 2015. 7: p. 33.

64. Vanden Berghe, W., et al., Keeping up NF-kappaB appearances: epigenetic control of immunity or inflammation-triggered epigenetics. Biochem Pharmacol, 2006. 72(9): p. 1114-31.

65. Vanden Berghe, W., Epigenetic impact of dietary polyphenols in cancer chemoprevention: lifelong remodeling of our epigenomes. Pharmacol Res, 2012. 65(6): p. 565-76. 
66. Milagro, F.I., et al., Dietary factors, epigenetic modifications and obesity outcomes: progresses and perspectives. Mol Aspects Med, 2013. 34(4): p. 782-812.

67. Remely, M., et al., Therapeutic perspectives of epigenetically active nutrients. Br J Pharmacol, 2015. 172(11): p. 2756-68.

68. Koch, A., et al., Analysis of DNA methylation in cancer: location revisited. Nat Rev Clin Oncol, 2018. 15(7): p. 459-466.

69. Milenkovic, D., et al., (-)-Epicatechin metabolites promote vascular health through epigenetic reprogramming of endothelial-immune cell signaling and reversing systemic low-grade inflammation. Biochem Pharmacol, 2019: p. 113699.

70. Dayeh, T., et al., Genome-wide DNA methylation analysis of human pancreatic islets from type 2 diabetic and non-diabetic donors identifies candidate genes that influence insulin secretion. PLoS Genet, 2014. 10(3): p. e1004160.

71. Langie, S.A.S., et al., GLI2 promoter hypermethylation in saliva of children with a respiratory allergy. Clin Epigenetics, 2018. 10: p. 50.

72. Deaton, A.M., et al., Cell type-specific DNA methylation at intragenic CpG islands in the immune system. Genome Res, 2011. 21(7): p. 1074-86.

73. Pulloor, N.K., et al., Human genome-wide RNAi screen identifies an essential role for inositol pyrophosphates in Type-l interferon response. PLoS Pathog, 2014. 10(2): p. e1003981.

74. Kutateladze, T.G., Histone deacetylation: IP4 is an epigenetic coregulator. Nat Chem Biol, 2012. 8(3): p. 230-1.

75. Burton, A., et al., Inositol pyrophosphates regulate JMJD2C-dependent histone demethylation. Proc Natl Acad Sci U S A, 2013. 110(47): p. 18970-5.

76. Meng, H., et al., DNA methylation, its mediators and genome integrity. Int J Biol Sci, 2015. 11(5): p. 604-17.

77. Papin, C., et al., Combinatorial DNA methylation codes at repetitive elements. Genome Res, 2017. 27(6): p. 934-946.

78. Nanan, K.K., et al., Independence between pre-mRNA splicing and DNA methylation in an isogenic minigene resource. Nucleic Acids Res, 2017. 45(22): p. 12780-12797.

79. Jones, P.A., Functions of DNA methylation: islands, start sites, gene bodies and beyond. Nat Rev Genet, 2012. 13(7): p. 484-92.

80. Kim, D., et al., Population-dependent Intron Retention and DNA Methylation in Breast Cancer. Mol Cancer Res, 2018. 16(3): p. 461-469.

81. Mancini, R.J., et al., Directing the immune system with chemical compounds. ACS Chem Biol, 2014. 9(5): p. 1075-85.

82. Muralidharan, S. and P. Mandrekar, Cellular stress response and innate immune signaling: integrating pathways in host defense and inflammation. J Leukoc Biol, 2013. 94(6): p. 1167-84. 
83. Piasecka, A., N. Jedrzejczak-Rey, and P. Bednarek, Secondary metabolites in plant innate immunity: conserved function of divergent chemicals. New Phytol, 2015. 206(3): p. 948-64.

84. Kamada, R., et al., Interferon stimulation creates chromatin marks and establishes transcriptional memory. Proc Natl Acad Sci U S A, 2018. 115(39): p. E9162-E9171.

85. Howitz, K.T. and D.A. Sinclair, Xenohormesis: sensing the chemical cues of other species. Cell, 2008. 133(3): p. 387-91.

86. Pham, A.M., et al., PKR Transduces MDA5-Dependent Signals for Type I IFN Induction. PLoS Pathog, 2016. 12(3): p. e1005489.

87. Andrilenas, K.K., et al., DNA-binding landscape of IRF3, IRF5 and IRF7 dimers: implications for dimerspecific gene regulation. Nucleic Acids Res, 2018. 46(5): p. 2509-2520.

88. Takada, Y., et al., Genetic deletion of PKR abrogates TNF-induced activation of IkappaBalpha kinase, JNK, Akt and cell proliferation but potentiates p44/p42 MAPK and p38 MAPK activation. Oncogene, 2007. 26(8): p. 1201-12.

89. Sun, S.C., The non-canonical NF-kappaB pathway in immunity and inflammation. Nat Rev Immunol, 2017. 17(9): p. 545-558.

90. Roesler, J., et al., Application of purified polysaccharides from cell cultures of the plant Echinacea purpurea to test subjects mediates activation of the phagocyte system. Int $\mathrm{J}$ Immunopharmacol, 1991. 13(7): p. 931-41.

91. Sokol, C.L. and A.D. Luster, The chemokine system in innate immunity. Cold Spring Harb Perspect Biol, 2015. 7(5).

92. Yuan, J., et al., CXCL10 inhibits viral replication through recruitment of natural killer cells in coxsackievirus B3-induced myocarditis. Circ Res, 2009. 104(5): p. 628-38.

93. Freeman, B.E., et al., Cytokine-Mediated Activation of NK Cells during Viral Infection. J Virol, 2015. 89(15): p. 7922-31.

94. Lodoen, M.B. and L.L. Lanier, Natural killer cells as an initial defense against pathogens. Curr Opin Immunol, 2006. 18(4): p. 391-8.

95. Monlun, M., et al., Mitochondria as Molecular Platforms Integrating Multiple Innate Immune Signalings. J Mol Biol, 2017. 429(1): p. 1-13.

96. Yin, S.Y., et al., Stimulatory effect of Echinacea purpurea extract on the trafficking activity of mouse dendritic cells: revealed by genomic and proteomic analyses. BMC Genomics, 2010. 11: p. 612.

97. Sharma, M., J.T. Arnason, and J.B. Hudson, Echinacea extracts modulate the production of multiple transcription factors in uninfected cells and rhinovirus-infected cells. Phytother Res, 2006. 20(12): p. 1074-9.

98. Cech, N.B., et al., Echinacea and its alkylamides: effects on the influenza A-induced secretion of cytokines, chemokines, and PGE(2) from RAW 264.7 macrophage-like cells. Int Immunopharmacol, 2010. 10(10): p. 1268-78. 
99. Sharma, M., et al., Echinacea extracts modulate the pattern of chemokine and cytokine secretion in rhinovirus-infected and uninfected epithelial cells. Phytother Res, 2006. 20(2): p. 147-52.

100. Wu, Y.H., et al., Avocado (Persea americana) fruit extract (2R,4R)-1,2,4-trihydroxyheptadec-16-yne inhibits dengue virus replication via upregulation of NF-kappaB-dependent induction of antiviral interferon responses. Sci Rep, 2019. 9(1): p. 423.

101. Snyder, D.T., et al., Oral delivery of oligomeric procyanidins in Apple Poly (R) enhances type I IFN responses in vivo. J Leukoc Biol, 2014. 95(5): p. 841-847.

102. Raniga, K. and C. Liang, Interferons: Reprogramming the Metabolic Network against Viral Infection. Viruses, 2018. 10(1).

103. Zhang, Y., et al., The in vivo ISGylome links ISG15 to metabolic pathways and autophagy upon Listeria monocytogenes infection. Nat Commun, 2019. 10(1): p. 5383.

104. Albert, M., et al., ISG15, a Small Molecule with Huge Implications: Regulation of Mitochondrial Homeostasis. Viruses, 2018. 10(11).

105. O'Neill, L.A., R.J. Kishton, and J. Rathmell, A guide to immunometabolism for immunologists. Nat Rev Immunol, 2016. 16(9): p. 553-65.

106. Viola, A., et al., The Metabolic Signature of Macrophage Responses. Front Immunol, 2019. 10: p. 1462.

107. Kumar, S. and M. Dikshit, Metabolic Insight of Neutrophils in Health and Disease. Front Immunol, 2019. 10: p. 2099.

108. Azevedo, E.P., et al., A Metabolic Shift toward Pentose Phosphate Pathway Is Necessary for Amyloid Fibril- and Phorbol 12-Myristate 13-Acetate-induced Neutrophil Extracellular Trap (NET) Formation. J Biol Chem, 2015. 290(36): p. 22174-83.

109. Takahashi-Tezuka, M., et al., Tec tyrosine kinase links the cytokine receptors to Pl-3 kinase probably through JAK. Oncogene, 1997. 14(19): p. 2273-82.

110. Yamashita, Y., et al., Tec and Jak2 kinases cooperate to mediate cytokine-driven activation of c-fos transcription. Blood, 1998. 91(5): p. 1496-507.

111. Lee, S. and S. Vasudevan, JNK MAPK Regulates IFN-Stimulated Genes and Cell Adhesion in Chemoresistant, Quiescent Leukemic Cells. BioRxiv, 2019. doi.org/10.1101/689570.

112. Tampella, G., et al., The Tec Kinase-Regulated Phosphoproteome Reveals a Mechanism for the Regulation of Inhibitory Signals in Murine Macrophages. J Immunol, 2015. 195(1): p. 246-56.

113. Zwolanek, F., et al., The non-receptor tyrosine kinase Tec controls assembly and activity of the noncanonical caspase-8 inflammasome. PLoS Pathog, 2014. 10(12): p. e1004525.

114. Melcher, M., et al., Essential roles for the Tec family kinases Tec and Btk in M-CSF receptor signaling pathways that regulate macrophage survival. J Immunol, 2008. 180(12): p. 8048-56.

115. Wang, G.Q., et al., Tec kinase mediating IL-8 transcription in monocytes stimulated with LPS. Inflammation, 2009. 32(4): p. 265-9. 
116. Jongstra-Bilen, J., et al., Dual functions of Bruton's tyrosine kinase and Tec kinase during Fcgamma receptor-induced signaling and phagocytosis. J Immunol, 2008. 181(1): p. 288-98.

117. Wang, F., et al., Inhibitor of Tec kinase, LFM-A13, decreases pro-inflammatory mediators production in LPS-stimulated RAW264.7 macrophages via NF-kappaB pathway. Oncotarget, 2017. 8(21): p. 3409934110.

118. Mikkelsen, S.S., et al., RIG-I-mediated activation of $p 38$ MAPK is essential for viral induction of interferon and activation of dendritic cells: dependence on TRAF2 and TAK1. J Biol Chem, 2009. 284(16): p. 10774-82.

119. Wang, C.Y., et al., Genomics and proteomics of immune modulatory effects of a butanol fraction of echinacea purpurea in human dendritic cells. BMC Genomics, 2008. 9: p. 479.

120. Li, Y., et al., Echinacea pupurea extracts promote murine dendritic cell maturation by activation of JNK, p38 MAPK and NF-kappaB pathways. Dev Comp Immunol, 2017. 73: p. 21-26.

121. Sullivan, A.M., et al., Echinacea-induced macrophage activation. Immunopharmacol Immunotoxicol, 2008. 30(3): p. 553-74.

122. Fu, A., et al., Echinacea purpurea Extract Polarizes M1 Macrophages in Murine Bone Marrow-Derived Macrophages Through the Activation of JNK. J Cell Biochem, 2017. 118(9): p. 2664-2671.

123. Yu, J.S., et al., Celastrol inhibits dengue virus replication via up-regulating type I interferon and downstream interferon-stimulated responses. Antiviral Res, 2017. 137: p. 49-57.

124. Brocks, D., et al., DNMT and HDAC inhibitors induce cryptic transcription start sites encoded in long terminal repeats. Nat Genet, 2017. 49(7): p. 1052-1060.

125. Shayevitch, R., et al., The importance of DNA methylation of exons on alternative splicing. RNA, 2018. 24(10): p. 1351-1362.

126. Greenberg, M.V.C. and D. Bourc'his, The diverse roles of DNA methylation in mammalian development and disease. Nat Rev Mol Cell Biol, 2019. 20(10): p. 590-607.

127. Savan, R., Alternative Splicing in Innate Antiviral Immunity. J Interferon Cytokine Res, 2018. 38(8): p. 317-318.

128. Chiappinelli, K.B., et al., Inhibiting DNA Methylation Causes an Interferon Response in Cancer via dsRNA Including Endogenous Retroviruses. Cell, 2015. 162(5): p. 974-86.

129. Yu, Q., et al., Type I interferon controls propagation of long interspersed element-1. J Biol Chem, 2015. 290(16): p. 10191-9.

130. Leonova, K.I., et al., p53 cooperates with DNA methylation and a suicidal interferon response to maintain epigenetic silencing of repeats and noncoding RNAs. Proc Natl Acad Sci U S A, 2013. 110(1): p. E89-98.

131. Licht, J.D., DNA Methylation Inhibitors in Cancer Therapy: The Immunity Dimension. Cell, 2015. 162(5): p. 938-9.

132. Grandi, N. and E. Tramontano, Human Endogenous Retroviruses Are Ancient Acquired Elements Still Shaping Innate Immune Responses. Front Immunol, 2018. 9: p. 2039. 
133. Buzdin, A.A., V. Prassolov, and A.V. Garazha, Friends-Enemies: Endogenous Retroviruses Are Major Transcriptional Regulators of Human DNA. Front Chem, 2017. 5: p. 35.

134. Sun, X., et al., Transcription factor profiling reveals molecular choreography and key regulators of human retrotransposon expression. Proc Natl Acad Sci U S A, 2018. 115(24): p. E5526-E5535.

135. Johnson, W.E., Origins and evolutionary consequences of ancient endogenous retroviruses. Nat Rev Microbiol, 2019. 17(6): p. 355-370.

136. Tokuyama, M., et al., ERVmap analysis reveals genome-wide transcription of human endogenous retroviruses. Proc Natl Acad Sci U S A, 2018. 115(50): p. 12565-12572.

137. Ito, J., et al., Systematic identification and characterization of regulatory elements derived from human endogenous retroviruses. PLoS Genet, 2017. 13(7): p. e1006883.

138. Hurst, T.P. and G. Magiorkinis, Activation of the innate immune response by endogenous retroviruses. J Gen Virol, 2015. 96(Pt 6): p. 1207-18.

139. Briard, B., D.E. Place, and T.D. Kanneganti, DNA Sensing in the Innate Immune Response. Physiology (Bethesda), 2020. 35(2): p. 112-124.

140. Canadas, I., et al., Tumor innate immunity primed by specific interferon-stimulated endogenous retroviruses. Nat Med, 2018. 24(8): p. 1143-1150.

141. Stone, O.A., et al., Loss of pyruvate kinase $M 2$ limits growth and triggers innate immune signaling in endothelial cells. Nat Commun, 2018. 9(1): p. 4077.

142. Chuong, E.B., N.C. Elde, and C. Feschotte, Regulatory evolution of innate immunity through co-option of endogenous retroviruses. Science, 2016. 351(6277): p. 1083-7.

143. Meyer, T.J., et al., Endogenous Retroviruses: With Us and against Us. Front Chem, 2017. 5: p. 23.

144. Hudson, J. and S. Vimalanathan, Echinacea-A Source of Potent Antivirals for Respiratory Virus Infections. Pharmaceuticals, 2011. 4(7): p. 1019-1031.

145. Yi, L., et al., Small molecules blocking the entry of severe acute respiratory syndrome coronavirus into host cells. J Virol, 2004. 78(20): p. 11334-9.

146. Signer, J., et al., In vitro antiviral activity of Echinaforce ${ }^{\circledR}$, an Echinacea purpurea preparation, against common cold coronavirus 229E and highly pathogenic MERS-CoV and SARS-CoV. BMC Virology journal, 2020: p. DOI:10.21203/rs.2.24724/v2.

\section{Figures}


A

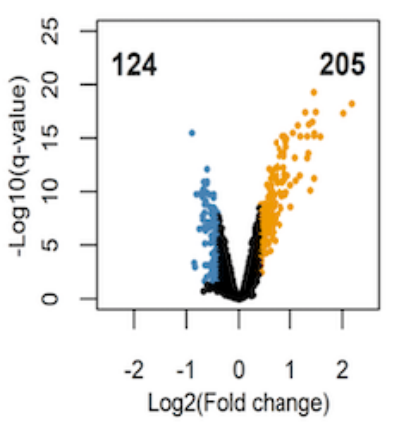

B

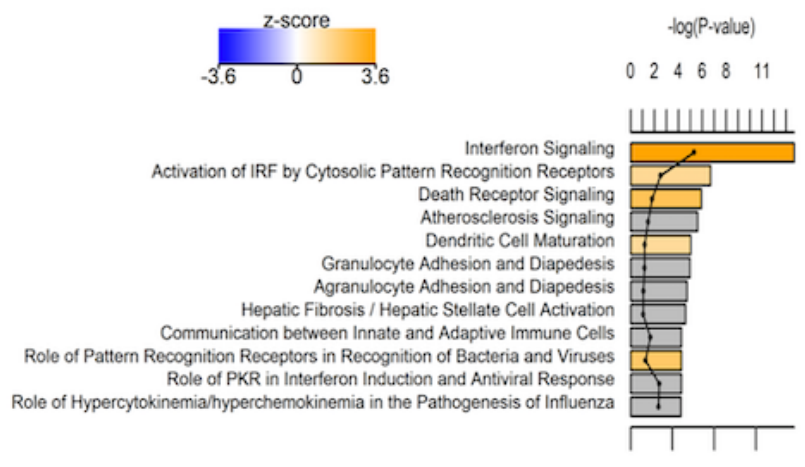

C

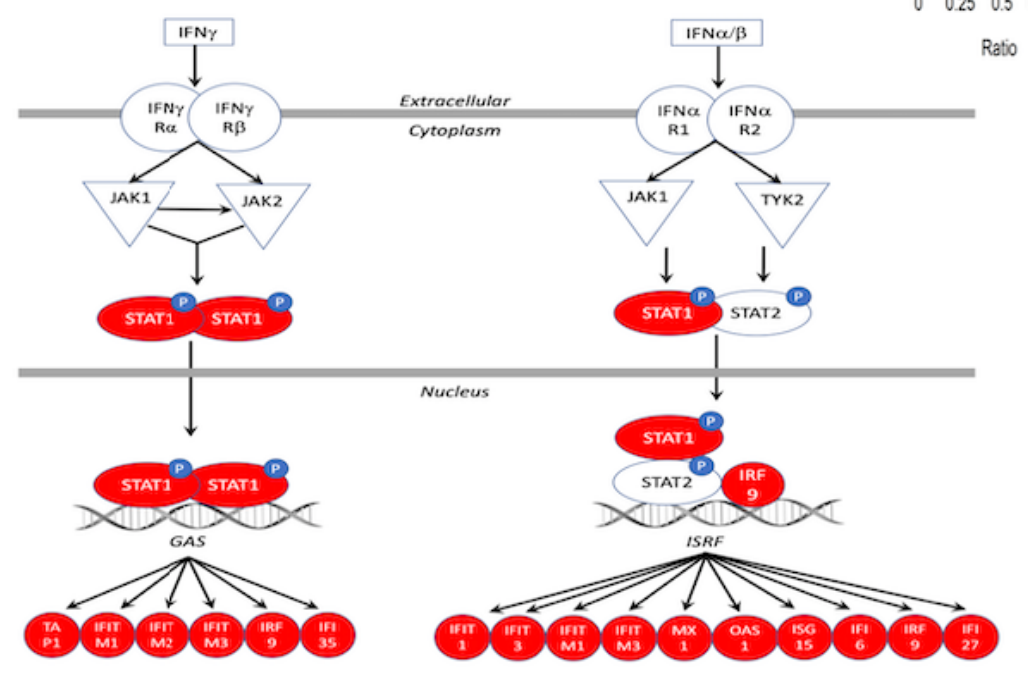

Ratio

D

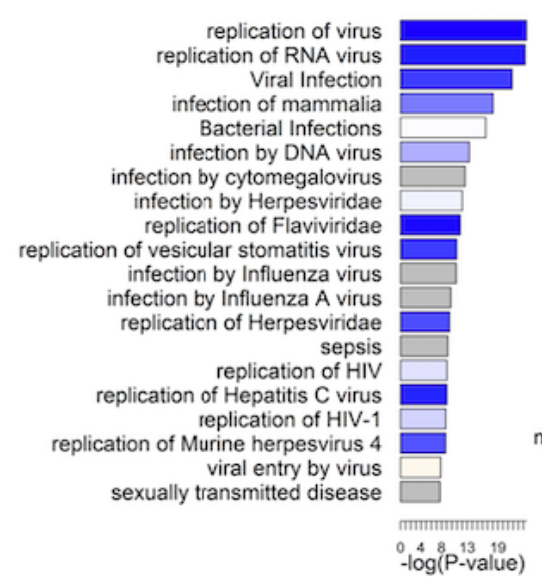

cellular infiltration by leukocytes cell movement of leukocytes adhesion of immune cells leukocyte migration cell movement of myeloid cells cell movement of phagocytes cell movement of neutrophils accumulation of leukocytes cell movement of granulocytes accumulation of myeloid cells recruitment of myeloid cells recruitment of myeloid cells recruitment of leukocytes accumulation of phagocytes homing of leukocytes recruitment of neutrophils accumulation of granulocytes recruitment of phagocytes movement of mononuclear leukocytes chemotaxis of leukocytes recruitment of granulocytes

${ }^{0}-\log ^{8}\left(P^{13}\right.$-value $)$

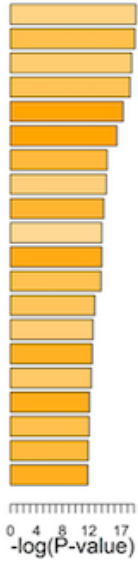

\section{Figure 1}

Echinaforce ${ }^{\circledR}$ induced gene expression activates innate immunity pathways A) Volcano plot showing the upregulated genes (orange color, number of probes: 205), and downregulated genes (blue color, number of probes: 124) upon treatment of THP1 cells for 48h with Echinaforce ${ }^{\circledR}$ tincture (1\%) . B) Top enriched IPA canonical pathways. Bars are colored by activation z-score. C) IPA interferon signaling pathway with Echinaforce ${ }^{\circledR}$-induced upregulated genes colored in red and green, respectively. D) Top enriched IPA 
infectious diseases and IPA immune trafficking disease and biological function. Bar charts are colored by activation z-score.

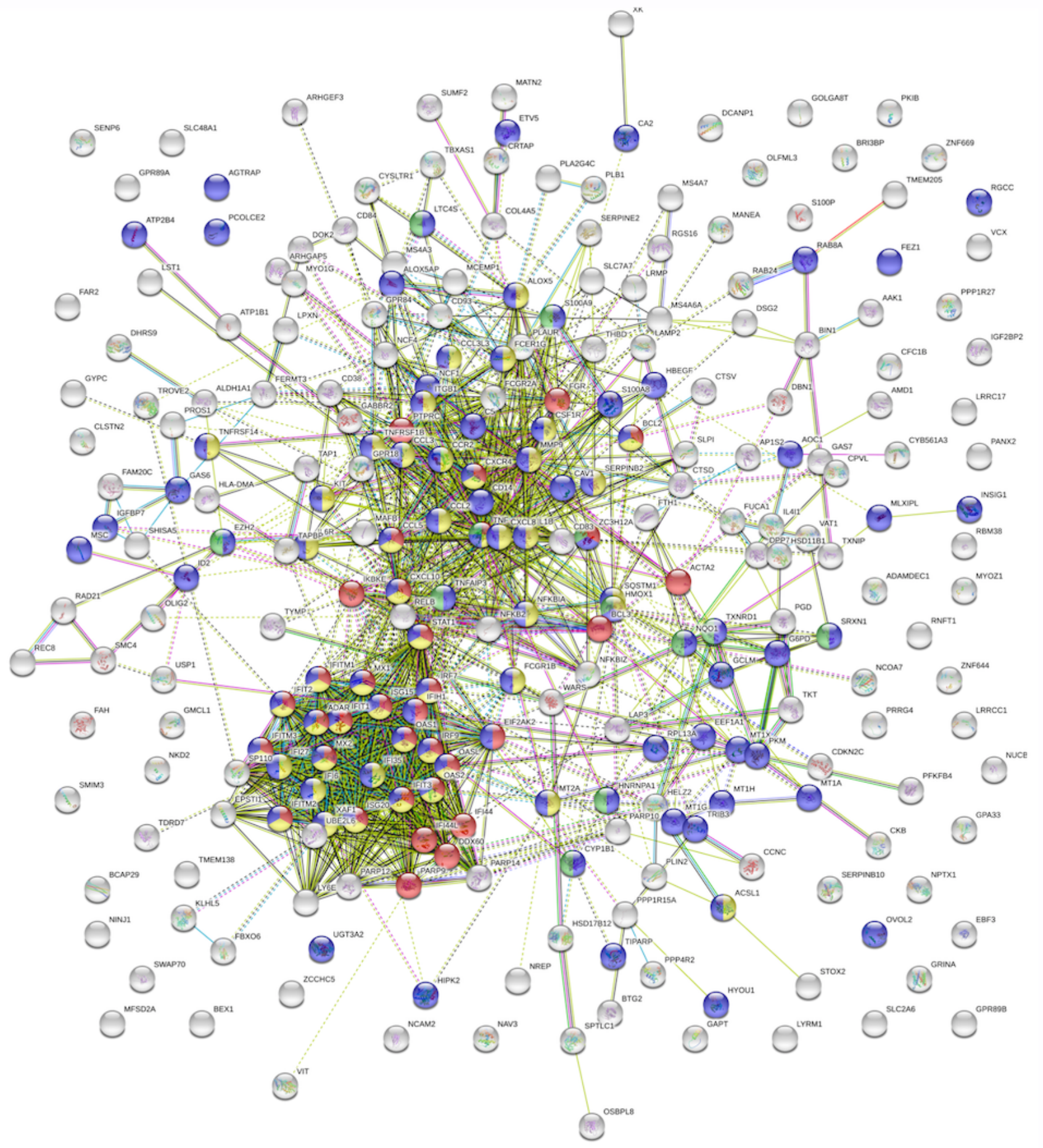

Figure 2

Protein-protein-interaction network analysis of Echinaforce ${ }^{\circledR}$ treatment responsive genes. STRING based protein-protein-interaction network analysis of differentially expressed genes of THP1 cells treated for 48h with Echinaforce ${ }^{\circledR}$ tincture (1\%) shows a strong network overlap of the cellular response to a 
chemical stimulus (FDR 2,91 E-18), cellular defense to virus (FDR 5,54 E-19) and innate immune cytokine response (FDR 2,23 E-18) (see also, supplementary table 4).

A
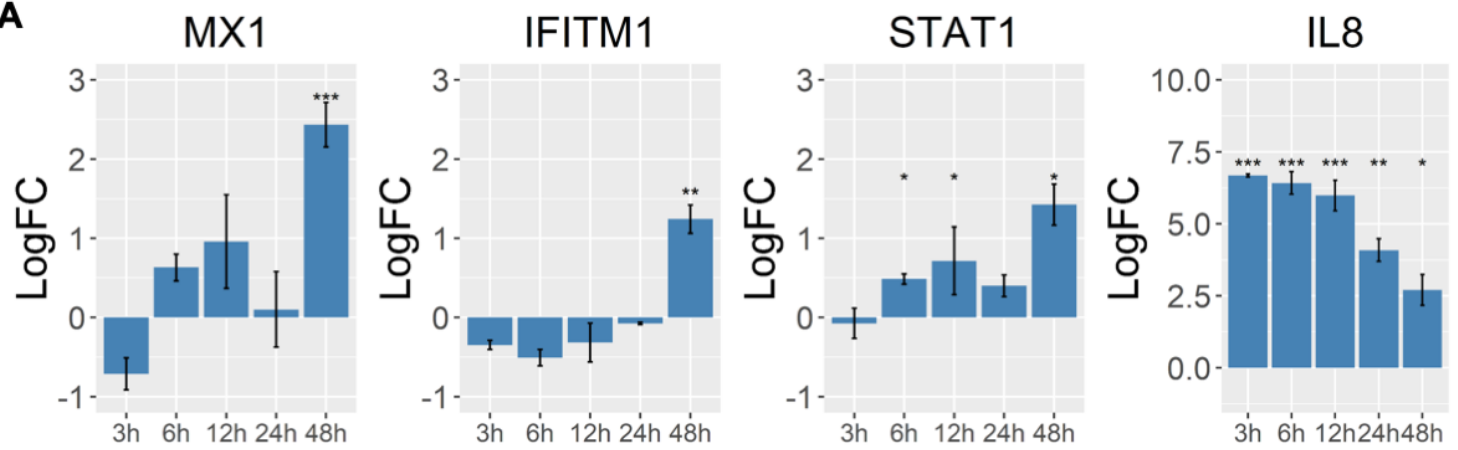

B
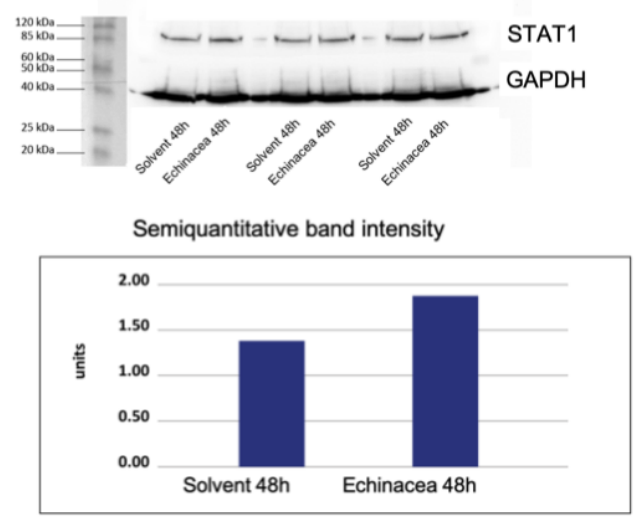

C



CXCL-10
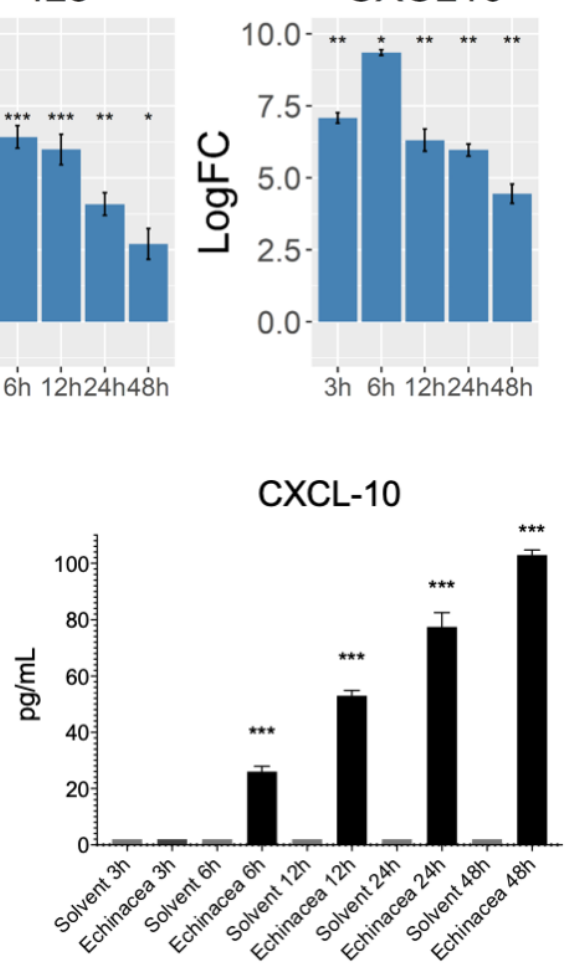

\section{Figure 3}

Induction of innate immune response by Echinaforce ${ }^{\circledR}$ represented by $A$ ) increased transcript levels of MX1, IFITM1, STAT1, IL -8 and CXCL10 genes at different time points, the bars represent the mean logFC


0.0001 . B) Protein levels of STAT1 and GAPDH (as reference protein) in $20 \mu \mathrm{g}$ protein cell lysates after 48 hours stimulation with solvent (Ethanol) and Echinaforce $\AA$; band intensities were determined using image J software and represent the band intensity of STAT1 blots divided by the band intensity of GAPDH. C) Increased levels of IL-8 and CXCL10 chemokines assayed by ELISA in supernatants collected after Echinaforce ${ }^{\circledR}$ and solvent (Ethanol) stimulation. *: $P \leq 0.05,{ }^{* *} P: \leq 0.01, * \star * P: \leq 0.001$ and ${ }^{* \star * *} P$ : $\leq 0.0001$. 

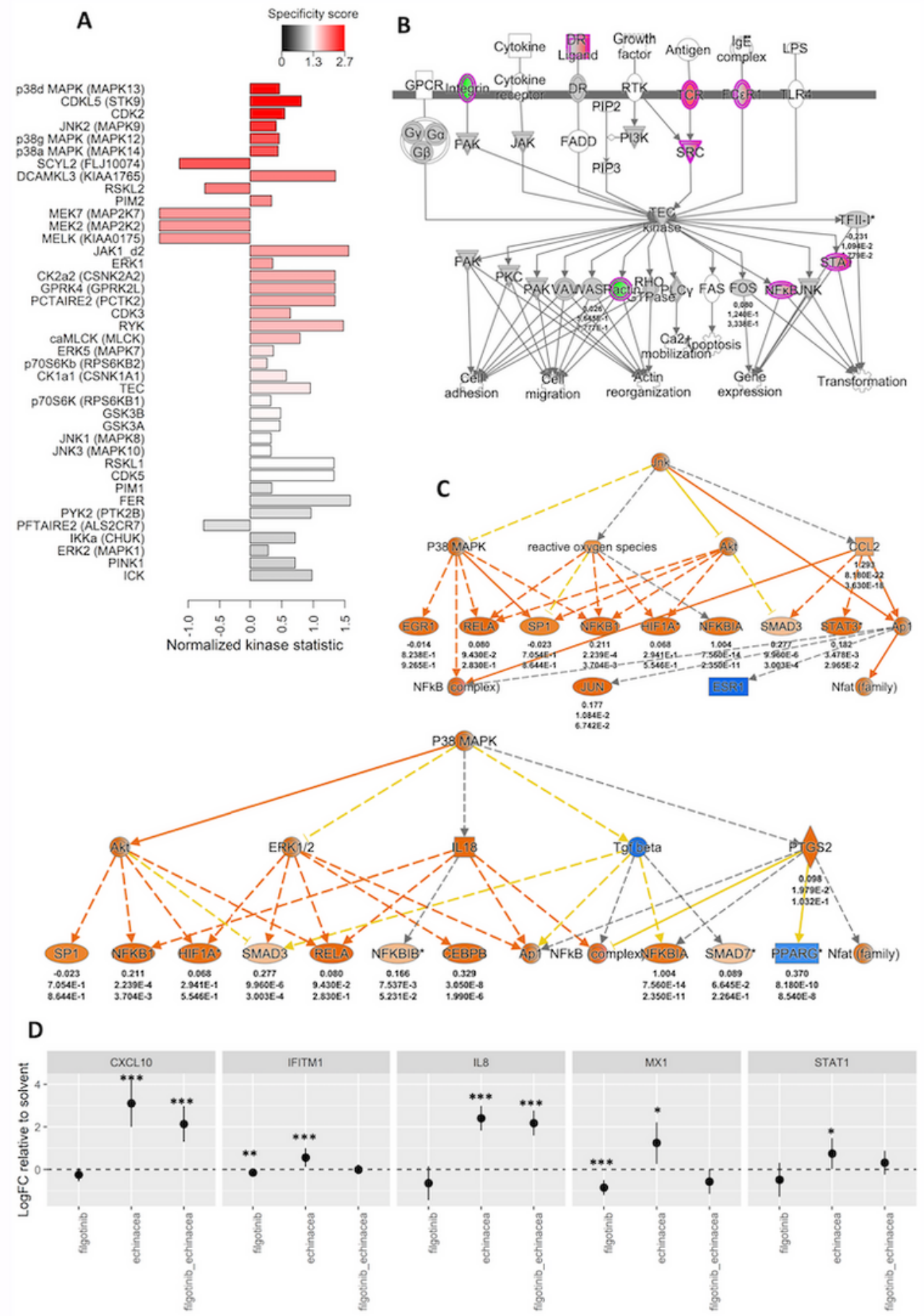

\section{Figure 4}

Activation of JAK1 and MAPK kinases by Echinaforce ${ }^{\circledR}$. A) Kinome activity profiling on THP1 cell lysates, following 15 min treatment with Echinaforce ${ }^{\circledR}$ tincture (1\%). Showing predicted upstream kinases. Bars are colored by specificity score with red meaning the highest score. The direction of the bars represents the normalized kinase statistics. A positive kinase statistic means a higher activity in Echinaforce ${ }^{\circledR}$ treated samples. B) TEC signaling pathway as predicted by IPA software showing the up- and down- 
regulated genes (colored in red and green, respectively), after Echinaforce ${ }^{\circledR}$ treatment. C) IPA-enriched P38 MAPK and JNK pathways upstream regulators. Genes colored in orange are predicted to be activated, while genes colored in blue are predicted to be inhibited. D) Effect of JAK1 inhibition on transcript expression of interferon pathway related genes. THP1 cells were either treated with the JAK1 inhibitor Filgotinib alone or in combination with Echinaforce ${ }^{\circledR}(n=7)$. Mean expression LogFC change relative to solvent control is represented together with $95 \%$ confidence interval. *: $P \leq 0.05$, ** $P$ : $\leq 0.01$, $\star \star \star P: \leq 0.001$.



\section{Figure 5}

Echinaforce ${ }^{\circledR}$ treatment leads to global hypermethylation of CpG-poor gene bodies. A) Heatmap showing the methylation values of differentially methylated probes upon treatment of THP1 cells for 48h with Echinaforce ${ }^{\circledR}$ tincture (1\%). Solvent $(\mathrm{EtOH})$ controls are colored in blue and Echinaforce ${ }^{\circledR}$-treated cells in orange. B) Genomic enrichment of DMPs in different genomic regions. C) CpG probes located in genes of the interferon signaling pathway which were differentially methylated (FDR $<0.1)$. $P \leq 0.05$, ** $P \leq 0.01$, $\star \star \star * P \leq 0.001, * \star \star \star P \leq 0.0001$. D) Starburst plot showing the genes both differentially expressed and differentially methylated. Each CpG-probe was mapped to its corresponding gene and the -log10(FDR) from the gene expression and DNA methylation analysis is displayed. The - log10(FDR) values of genes or CpG-probes with a negative LogFC or delta beta was multiplied by -1 leading to positive values when logFC or delta beta was positive and negative values when logFC or delta beta was negative. CpG-probe - gene pairs which were differentially expressed (FDR $<0.05)$ and differentially methylated (FDR $<0.1)$ were colored in blue. The CpG-probe - gene pairs of which the absolute delta beta was higher than 0.05 and the absolute logFC higher than 0.4 were colored in red. E) The IPA canonical pathways which were both significantly enriched in the gene expression and DNA methylation analysis.
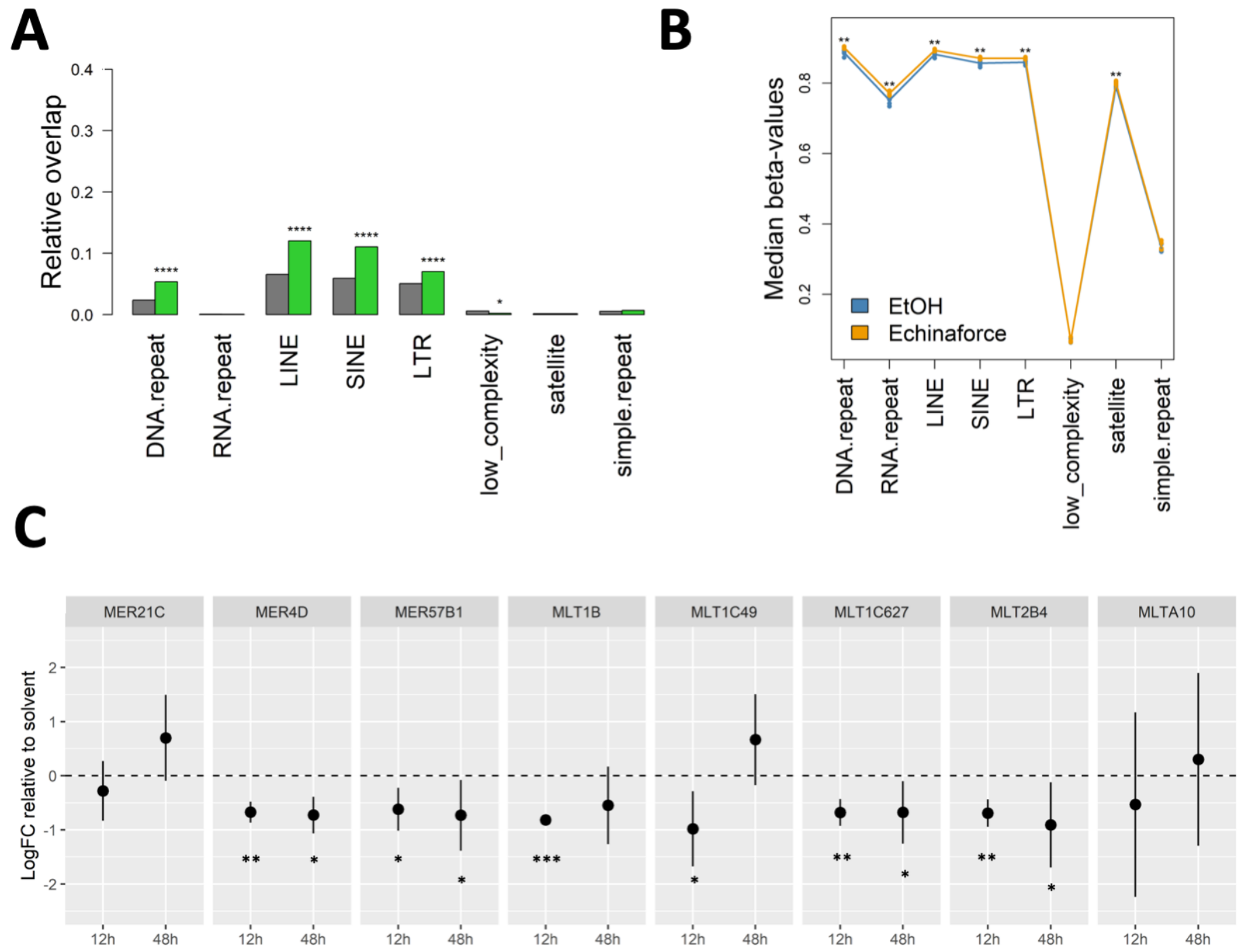


\section{Figure 6}

Echinaforce ${ }^{\circledR}$ treatment leads to global hypermethylation of intergenic repeat elements. A) Genomic enrichment of DMPs in different repeat elements. B) Global DNA methylation changes in different repeat elements. C) HERV qPCR gene expression. THP1 cells were with Echinaforce ${ }^{\circledR}$ at 12 and $48 \mathrm{~h}(\mathrm{n}=3)$. Mean LogFC change relative to solvent control is represented together with $95 \%$ confidence interval. $*$ : $P \leq 0.05$, ** $\mathrm{P}: \leq 0.01, * * * \mathrm{P}: \leq 0.001$ and $* * * * \mathrm{P}: \leq 0.0001$.

\section{Supplementary Files}

This is a list of supplementary files associated with this preprint. Click to download.

- Supplementarytable4.xlsx

- Supplementarytable2.xlsx

- Supplementarytable1.xlsx

- Supplementarytable6.xlsx

- Supplementarytable3.xIsx

- Supplementarytable5.xlsx

- Supplementarytable7.xlsx 